\title{
Influenza epidemiology and influenza vaccine effectiveness during the 2016-2017 season in the Global Influenza Hospital Surveillance Network (GIHSN)
}

Víctor Baselga-Moreno ${ }^{1}$, Svetlana Trushakova², Shelly McNeil ${ }^{3}$, Anna Sominina ${ }^{4}$, Marta C. Nunes ${ }^{5,6}$, Anca Draganescu', Serhat Unal ${ }^{8}$, Parvaiz Koul ${ }^{9}$, Jan Kyncl ${ }^{10}$, Tao Zhang ${ }^{11}$, Ainagul Kuatbayeva ${ }^{12}$, Afif Ben-Salah ${ }^{13,14}$, Elena Burtseva ${ }^{2}$, Joan Puig-Barberà', Javier Díez-Domingo ${ }^{1 *}$ and for the Global Influenza Hospital Surveillance Network (GIHSN)

\begin{abstract}
Background: The Global Influenza Hospital Surveillance Network (GIHSN) aims to determine the burden of severe influenza disease and Influenza Vaccine Effectiveness (IVE). This is a prospective, active surveillance and hospitalbased epidemiological study to collect epidemiological data in the GIHSN. In the 2016-2017 influenza season, 15 sites in 14 countries participated in the GIHSN, although the analyses could not be performed in 2 sites. A common core protocol was used in order to make results comparable. Here we present the results of the GIHSN 2016-2017 influenza season.

Methods: A RT-PCR test was performed to all patients that accomplished the requirements detailed on a common core protocol. Patients admitted were included in the study after signing the informed consent, if they were residents, not institutionalised, not discharged in the previous 30 days from other hospitalisation with symptoms onset within the 7 days prior to admission. Patients 5 years old or more must also complied the Influenza-Like IIIness definition. A test negative-design was implemented to perform IVE analysis. IVE was estimated using a logistic regression model, with the formula IVE $=(1-\mathrm{aOR}) \times 100$, where aOR is the adjusted Odds Ratio comparing cases and controls.
\end{abstract}

Results: Among 21,967 screened patients, 10,140 (46.16\%) were included, as they accomplished the inclusion criteria, and tested, and therefore 11,827 (53.84\%) patients were excluded. Around $60 \%$ of all patients included with laboratory results were recruited at 3 sites. The predominant strain was $A(H 3 N 2)$, detected in $63.6 \%$ of the cases (1840 patients), followed by BNictoria, in 21.3\% of the cases (618 patients). There were 2895 influenza positive patients (28.6\% of the included patients). A(H1N1)pdm09 strain was mainly found in Mexico. IVE could only be performed in 6 sites separately. Overall IVE was 27.24 (95\% Cl 15.62-37.27. Vaccination seemed to confer better protection against influenza $B$ and in people $2-4$ years, or 85 years old or older. The aOR for hospitalized and testing positive for influenza was 3.02 (95\% Cl 1.59-5.76) comparing pregnant with non-pregnant women.

Conclusions: Vaccination prevented around 1 in 4 hospitalisations with influenza. Sparse numbers didn't allow estimating IVE in all sites separately. Pregnancy was found a risk factor for influenza, having 3 times more risk of being admitted with influenza for pregnant women.

Keywords: Influenza virus, Surveillance, Vaccine effectiveness, Epidemiology

\footnotetext{
* Correspondence: jdiezdomingo@gmail.com

${ }^{1}$ Fundación para el Fomento de la Investigación Sanitaria y Biomédica de la

Comunidad Valenciana (FISABIO), 21 Cataluña Av, 46020 Valencia, Spain

Full list of author information is available at the end of the article
}

(c) The Author(s). 2019 Open Access This article is distributed under the terms of the Creative Commons Attribution 4.0 International License (http://creativecommons.org/licenses/by/4.0/), which permits unrestricted use, distribution, and

reproduction in any medium, provided you give appropriate credit to the original author(s) and the source, provide a link to the Creative Commons license, and indicate if changes were made. The Creative Commons Public Domain Dedication waiver (http://creativecommons.org/publicdomain/zero/1.0/) applies to the data made available in this article, unless otherwise stated. 


\section{Background}

Influenza is a major public health problem that can cause hospitalisations, and it is related with respiratory failures $[1,2]$. The Global Influenza Hospital Surveillance Network (GIHSN) is an international public-private collaboration that started in 2012. The GIHSN goals are to improve understanding of influenza epidemiology, quantifying the circulation of the different types and subtypes of influenza, in order to measure the effectiveness of seasonal influenza vaccines and better inform public health policy decisions. We conduct a prospective, active surveillance, hospital-based epidemiological study that collects epidemiological and virological data from those sites that are included in the network. Each season results are presented in annual meetings and, since 2012, have been published [3-6], with the agreement of the Principal Investigators of all concerned sites. The implementation and data collection for the last season (2016-2017) was led by the Foundation for the Promotion of Health and Biomedical Research of Valencia Region (FISABIO), a regional public health institution in Valencia, Spain, and funded by the Foundation for Influenza Epidemiology. Fifteen sites in fourteen countries participated in the GIHSN in the season 2016-2017. Among them, there were 12 sites (St. Petersburg, Moscow, Kazakhstan, Czech Rep., Canada, Romania, Turkey, Spain, Tunisia, Suzhou/Shanghai, India and Mexico) from Northern Hemisphere countries not situated in the tropics and three sites (Ivory Coast, Peru and South Africa) from the tropics or the Southern Hemisphere. Since Peru and Ivory Coast only reported two positive cases for influenza in the influenza season, the analysis was performed without the data from these countries, and therefore, results are reported for 13 sites. A common core protocol and standard operating procedures are used for all participating sites, in order to allow comparisons among countries, and analyse results of all sites.

\section{Methods}

This study aims to determine the frequency of influenzarelated hospitalisations in different countries, by circulating strains and age groups, to study risk factors for influenza-associated hospitalisations and estimate Influenza Vaccine Effectiveness (IVE) by site, age group and strain. Each site had one or more hospitals that recruited patients for the study, between October 2016 and May 2017 in Northern Hemisphere sites, except China, whose patients were recruited between June and September. For Southern Hemisphere sites, patients were recruited between May and November. Patients were included in the study if they presented any of the admission diagnoses included in the protocol, and only if they signed the informed consent to participate in the study. Among them, we selected for the study only those who were residents in the predefined hospital catchment's area in the previous past 6 months, who were not institutionalised, who hadn't been discharged from other hospitalisation in the last 30 days, and who had symptoms possibly related to influenza in 7 days or less prior to admission (Fig. 1). We also excluded patients who had previously tested positive for influenza in the current season, and also patients for whom the difference between the date of the onset of symptoms and the date of swabbing was 10 days or more (that is, those admitted after the 7th day after the onset of symptoms+maximum delay in swabbing). For patients 5 years old or more, they must also have complied with the Influenza-Like Illness (ILI) definition, detailed in European Centre for Disease Prevention and Control (ECDC) protocols, according to the decision of the Commission of the European Union of 8 August 2012 [7]. Patients enrolled outside the influenza epidemic period of each of the participating sites were also excluded. Influenza seasons were previously determined by each site, following recommendations of previous studies [8]. This methodology has been used in the GIHSN since the beginning of the network, and has been previously described [9]. For patients under 14 years old, nasal and/or nasopharyngeal swabs were collected, whereas, for patients 14 years old or more, pharyngeal and/or nasopharyngeal swabs were taken. Reverse transcription-polymerase chain reaction (RT-PCR) was used, according to each site's protocol, in order to detect influenza virus; viral subtyping was performed in order to identify $\mathrm{A}(\mathrm{H} 1 \mathrm{~N} 1) \mathrm{pdm} 09, \mathrm{~A}(\mathrm{H} 3 \mathrm{~N} 2)$, B/Yamagata-lineage, and $\mathrm{B} /$ Victoria-lineage strains in the positive specimens.

We performed a test-negative study [10] in order to compare positives (cases) and negatives (controls) for influenza and estimate Influenza Vaccine Effectiveness (IVE). Odds Ratios were used to estimate IVE, comparing cases and controls of patients depending on the vaccination status. Patients were considered vaccinated if they received an influenza vaccine in the current season, at least 15 days before the onset of symptoms. Patients with contra-indication to influenza vaccination were excluded from the IVE analysis, but were included in the analysis regarding influenza circulation. Vaccination status was ascertained either by recall or by vaccination registries. Adjusted odds ratios (aOR) were calculated using a logistic regression model including sex, occupational social class, obesity status, pregnancy, underlying conditions, general practitioner (GP) consultations in last 3 months, smoking habits, days from onset of symptoms to swabbing as fixed effects, age and epidemiological week of admission using cubic splines, and site as a cluster variable, in order to consider sites variability [11]. IVE was calculated as $(1-\mathrm{aOR}) \times 100$. The same factors were used to adjust IVE by strain or age group. The variables relative to the Barthel Index (in patients 65 years old or older) and the previous hospitalisations in the last year were initially considered to be included in 


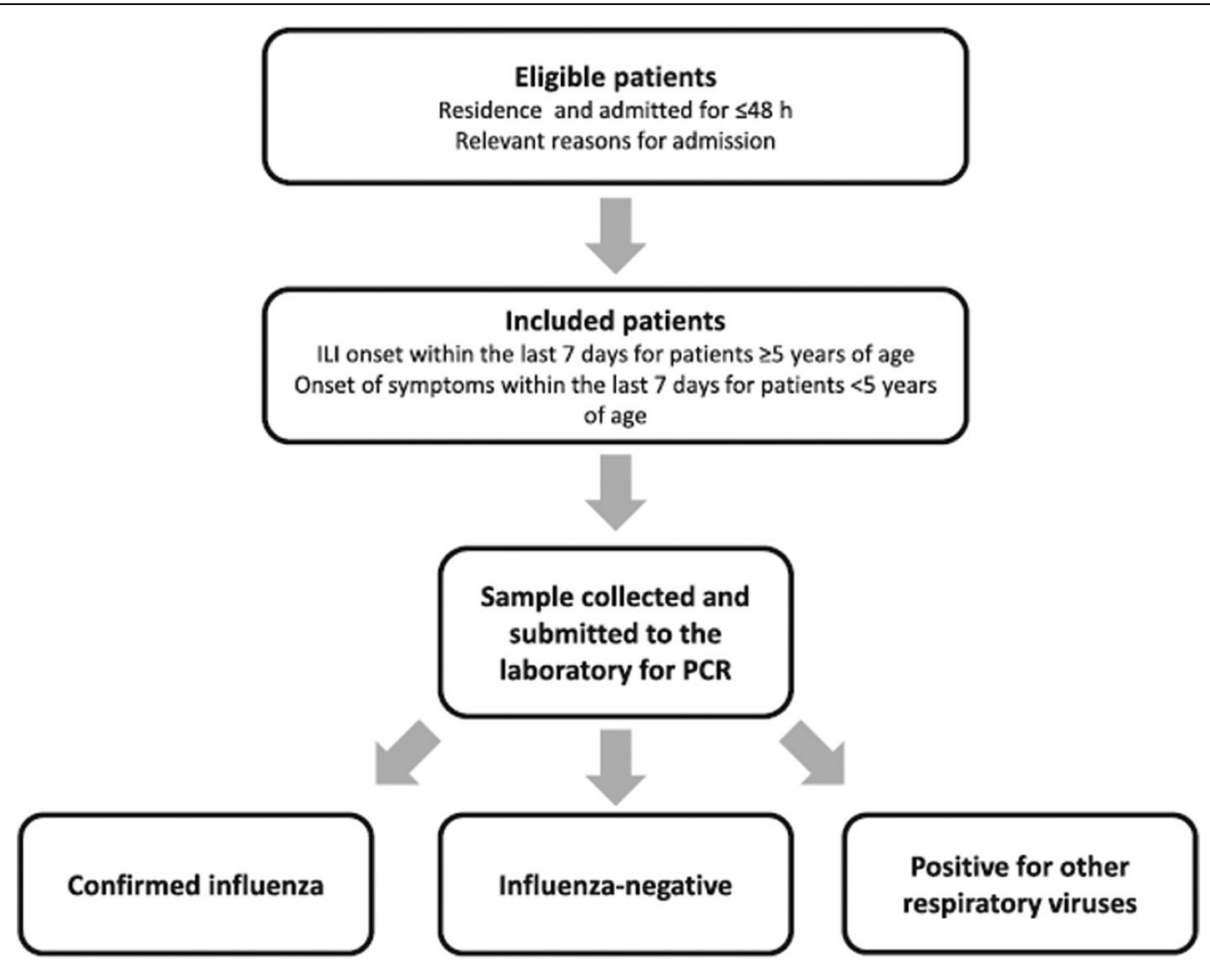

Fig. 1 Overview of the methodology used by the GIHSN

the model, but were excluded from the final model as they were not statistically significant considering all variables mentioned above. The model did not include the number of consultations at the GP in the last 3 months to estimate IVE in Canada, as this site did not provide information for this variable. Severe outcomes were also studied, defining them as an influenza positive patient admitted to ICU during the hospitalisation, or with COPD exacerbation, respiratory failure, any cardiovascular complication, shock or death during hospitalisation. Heterogeneity was studied, using the $\mathrm{I}^{2}$ test, and considering that heterogeneity was relevant if $\mathrm{I}^{2} \geq 50 \%[12,13]$.

\section{Results}

Included patients: distribution, characteristics and influenza positives and negatives

There were 21,967 eligible admissions between October 1, 2016 and November 9, 2017. However, only 10,140 patients complied with the conditions described above, and had laboratory results, hence only these were included in the analysis. Among them, 2895 (28.6\%) tested positive for influenza, and $7245(71.4 \%)$ tested negative for influenza (Table 1). The most common reason of exclusion was the fact that patients didn't have ILI symptoms in the 7 days previous to admission. It is important to note that $2 / 3$ of all included patients in the GIHSN came from 4 sites (St. Petersburg, Moscow, Canada and Valencia). These 4 sites also have the highest numbers of influenza positive cases, including $77.8 \%$ of all influenza positives in the GIHSN, and $84.3 \%$ of the $\mathrm{A}(\mathrm{H} 3 \mathrm{~N} 2)$ influenza positives among all participant sites. A (H3N2) was the predominant strain this season, being detected in $63.6 \%$ of all influenza positive cases (1840 patients), followed by $\mathrm{B} /$ Victoria, with $21.3 \%$ among the influenza positive cases (618 patients) (Table 1). Influenza A(H3N2) was detected throughout the season, whereas B/Victoria started to increase in the second week of 2017 in the Northern Hemisphere, and in the 31st week of 2017 in the Southern Hemisphere, approximately in the middle of the season in each Hemisphere (Fig. 2).

In the Northern Hemisphere, there was a significant increase in the number of influenza cases in week \#49 of 2016, with a peak in the number of positive cases during the second week of 2017 and starting to descend at the eighth week of 2017. Influenza B/Victoria started to increase clearly in the second week of 2017, as A(H3N2) started to descend. $70.3 \%$ of all influenza cases were positive for influenza A, whereas $29.7 \%$ were positive for influenza $\mathrm{B}$, with a clear different distribution among sites.

$\mathrm{A}(\mathrm{H} 3 \mathrm{~N} 2)$ was predominant in all sites, except in Mexico, where the predominant strain was $\mathrm{A}(\mathrm{H} 1 \mathrm{~N} 1) \mathrm{pdm} 09$, and Romania and India with a predominance of $\mathrm{B} /$ Victoria-lineage. Both $B$ lineages circulated during this season, with geographical differences, so in Canada, Czech Republic, Turkey, Tunisia, Mexico and South Africa, B/Yamagata was more often detected, while the B/Victoria was elsewhere. 


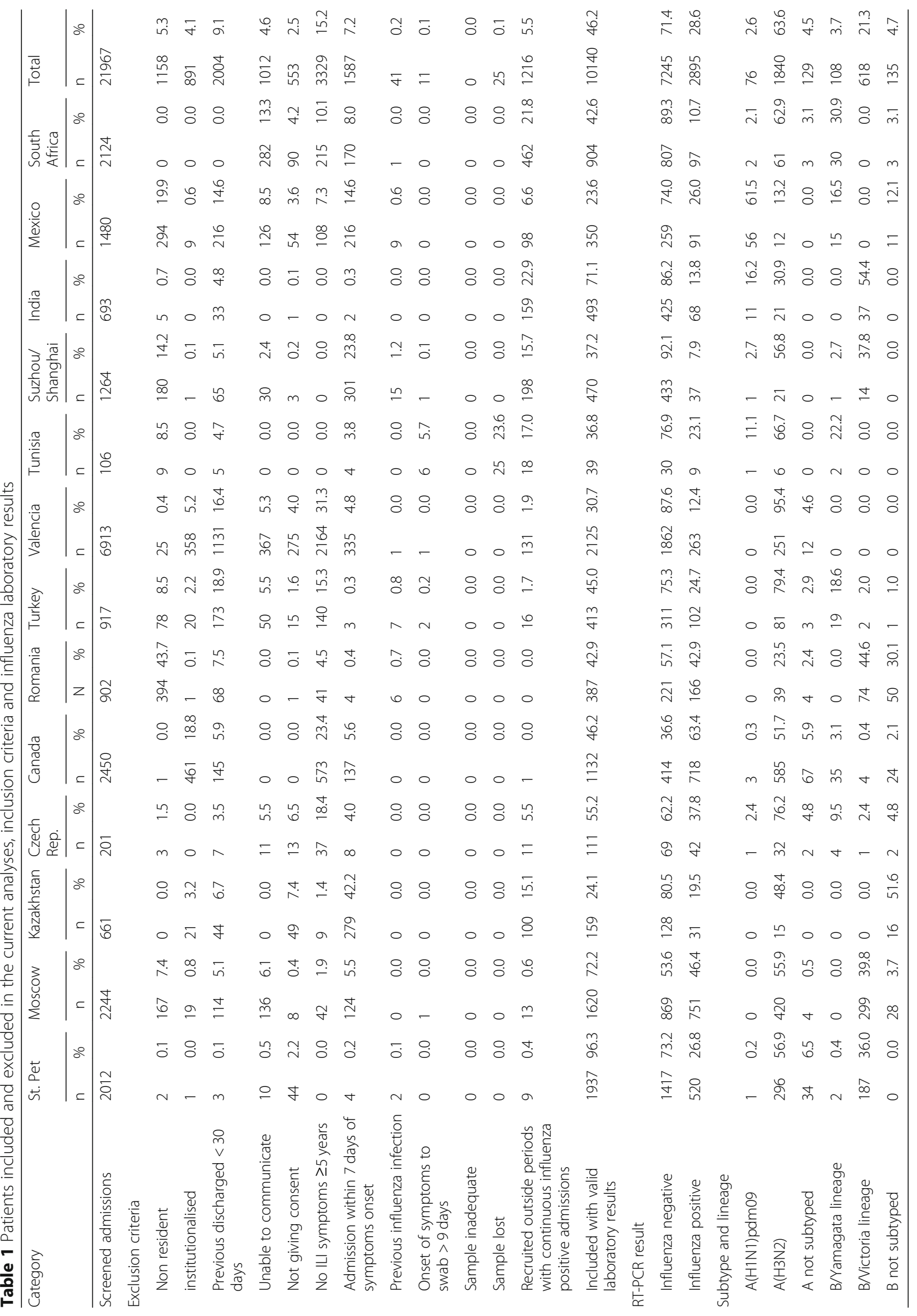




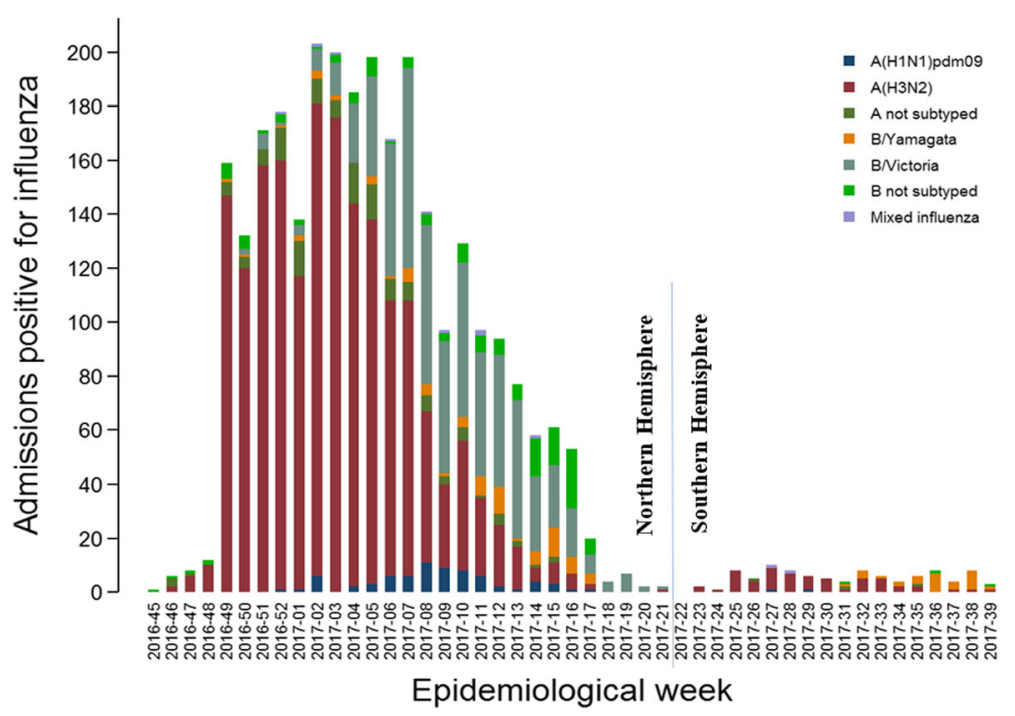

Fig. 2 Influenza-associated admissions by epidemiological week and virus type/subtype

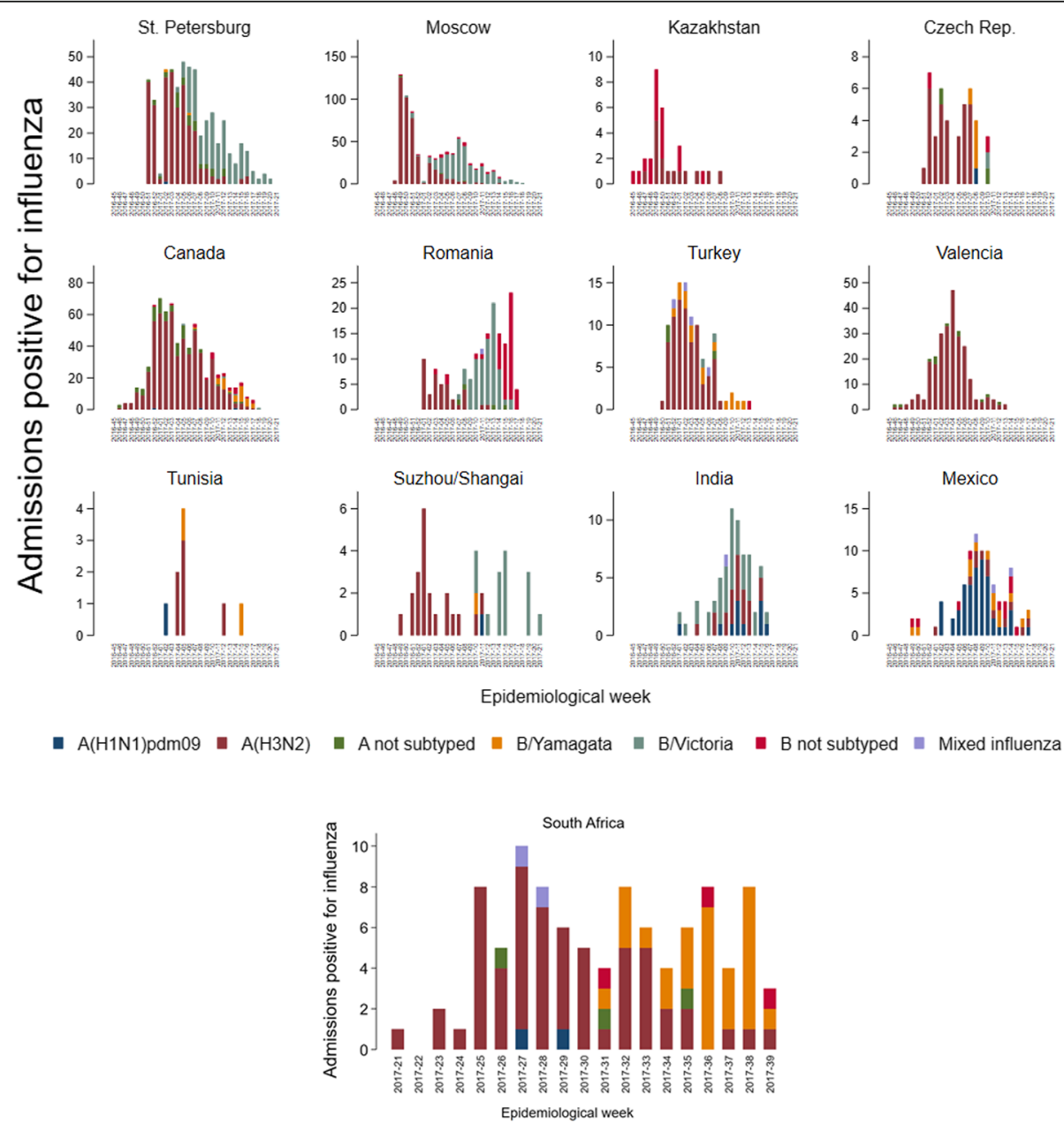

Fig. 3 Admissions with influenza by site, epidemiological week and virus type/subtype 
Influenza B cases generally appeared as a second influenza wave (Fig. 3). In Valencia, no cases were positive for influenza B.

Influenza B was mainly observed in the youngest, and was the predominant strain in the age group 5-17 years old. Among the two influenza B lineages, in general B/ Victoria was detected more often than B/Yamagata, except in the age group 50-64 years (Fig. 4).

The distribution of influenza cases among the age groups was clearly different among sites, but differences were mainly due to the characteristics of the participating hospitals for each site. Tunisia and Czech Republic only recruited patients 18 years old or older, while Suzhou/Shanghai only enrolled patients under 18 years old. In Moscow, the majority of influenza positives were pregnant women (which represented the $49.4 \%$ of the included patients), and therefore, the highest number of influenza positives among the different age groups was situated in the age group 18-49 years old in this site. Influenza positive cases were mainly found in patients 65 years old or older in Valencia and Canada, but $89.8 \%$ of the included patients from Canada were 50 years old or older. In St. Petersburg and South Africa, due to the characteristics of the patients of the participating hospitals (mainly children) there were more influenza positive cases in the youngest groups (Fig. 5).

$25.8 \%$ of the included patients were previously hospitalised in the same year and $36.6 \%$ of the included patients had at least one underlying condition, but this percentage varied among sites, in Canada, for example, more than $90 \%$ of the included patients had at least one underlying condition, whereas in St. Petersburg, this percentage was lower than $10 \%$ and in Turkey was $48.2 \%$, but these percentages could be related to the age distribution of the included patients in each site. Among the different comorbidities, the most common were cardiovascular $(20.7 \%$ of the included patients), diabetes $(10.4 \%)$ and chronic obstructive pulmonary disease (COPD) (9.9\%). Obesity was also found in more than $14 \%$ of the included patients, being more relevant in Canada (29.6\%), Valencia (26.3\%) and Czech Republic (23.4\%). Moscow was the site with the highest number of pregnant women among all sites (800 pregnant in Moscow among 940 pregnant women in all sites), being $49.4 \%$ of the included patients in this site. In Kazakhstan, pregnant women represented $22.6 \%$ of the included patients. The Barthel Index in those over 65 years showed that almost $90 \%$ of these subjects were not dependent or had a mild dependence. $68.3 \%$ of the patients who tested negative for influenza were swabbed from 0 to 4 days after symptoms started, but this percentage was $78.4 \%$ for influenza positive cases ( $p$-value $<0.0001$ ).

Vaccination coverage differed among sites. Patients were considered as vaccinated if vaccination was at least 15 days before symptoms onset (Table 2). Targeted patients for vaccination criteria were different among sites (Additional file 1: Complementary Table S1). Vaccination coverage was $11.1 \%$ among the influenza positives and $18.4 \%$ among the influenza negatives overall. Cardiovascular diseases, renal impairment, chronic obstructive pulmonary

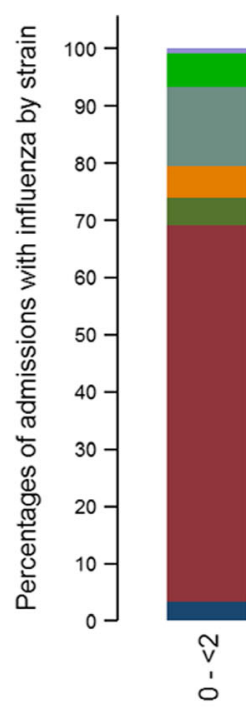

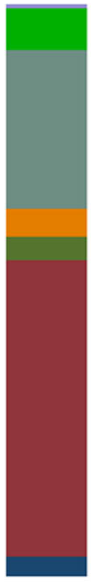

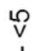

ì

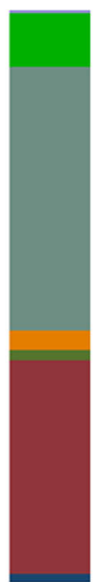

$\frac{\infty}{v}$
1
1

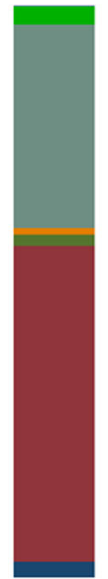

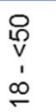
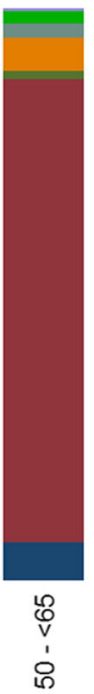

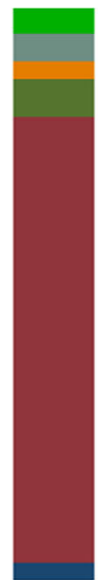

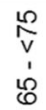
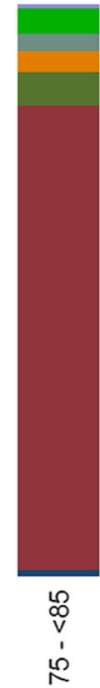

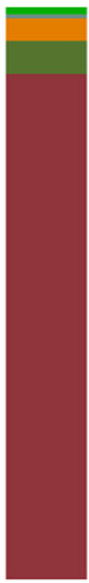

$\stackrel{\infty}{\infty}$

\section{Age group}

A(H1N1)pdm09

BNictoria

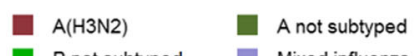

- B not subtyped Mixed influenza
B/Yamagata

Fig. 4 Percentages of influenza-associated admissions by age group and type/subtype 


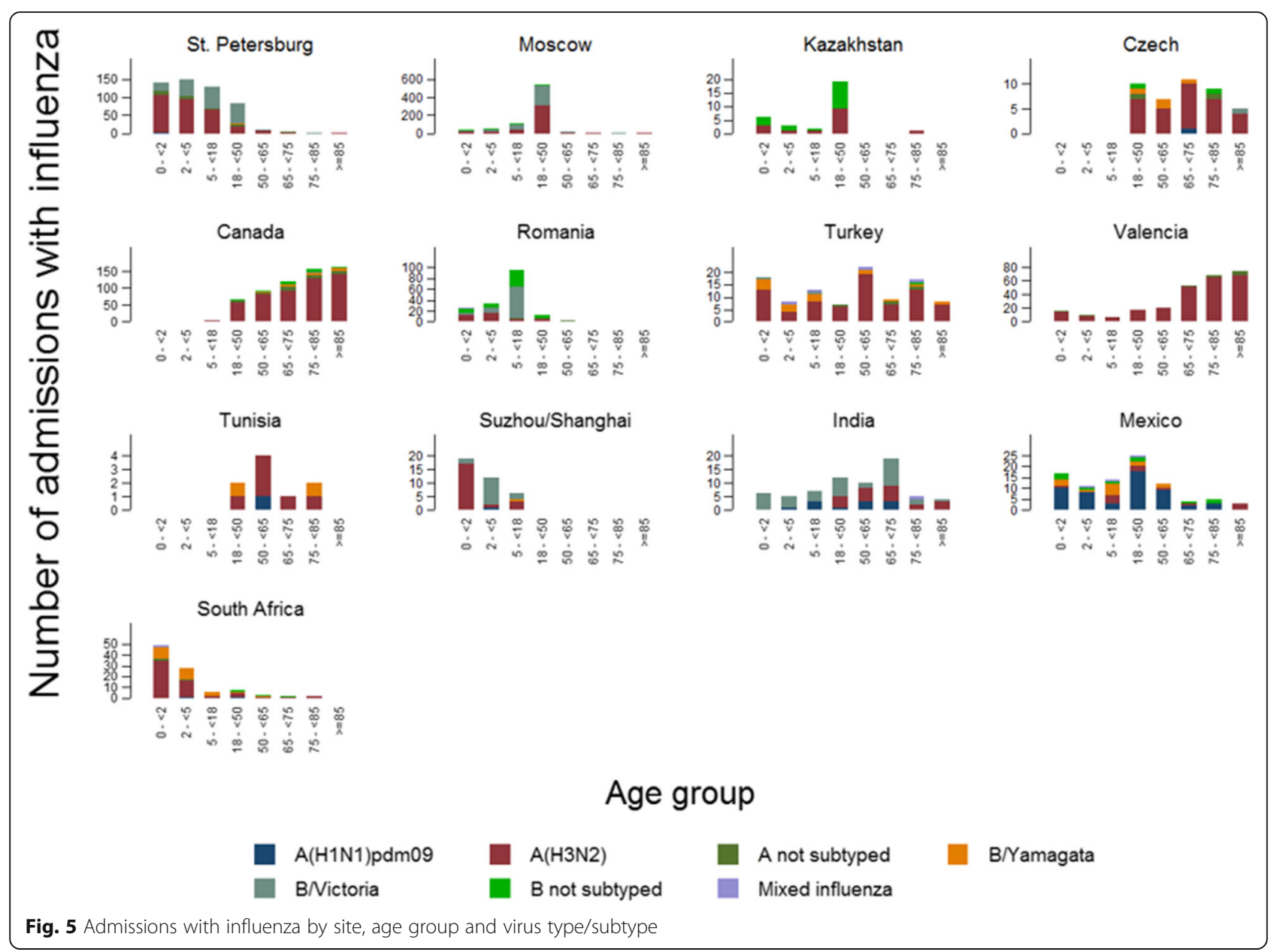

disease and diabetes were the most common comorbidities among influenza positives (Table 3). Seasonality had also a clear geographical distribution. Sites in higher latitudes had, generally, an earlier start of the influenza season.

Patients with a qualified occupation had a higher risk of being admitted with influenza. Patients with a swab taken 8-9 days after symptoms onset appeared with less risk of being admitted with influenza, suggesting a decrease in the influenza viral load for these patients (Table 4).

Pregnant women had a 3 times higher risk of having influenza at admission than non-pregnant. Also subjects with diabetes had 1.19 times higher risk of being an influenza case. On the other hand, patients with COPD or neoplasm had lower risk of testing positive for influenza. Despite there was a high number of admissions with cardiovascular diseases (CVD), no difference in the risk of influenza was found in these patients. (Fig. 6).

During pregnancy, the risk of testing positive for influenza was higher during the third trimester than in the first trimester, and also if they had any comorbidity in the first trimester (Fig. 7).
There were no significant statistical differences among influenza positives and negatives for those who were admitted to ICU or who received mechanical ventilation or those who died while they were hospitalised, and differences for those with extracorporeal membrane oxygenation could be due to sparse numbers of patients who received extracorporeal membrane oxygenation. Apart from influenza, the main discharge diagnosis was pneumonia, either for influenza-negatives or influenza-positives (Table 5).

Probabilities of most common severe outcomes by strain by age and influenza strains are displayed in Fig. 8. This probability had an upward trend up to 80 years old after a shock. The probability point estimates of having any cardiovascular complication increased greatly from 90 years old for those who had influenza. Similar trends were found for each individual strain for these discharge diagnoses.

Vaccination coverage was $9 \%$ or higher for targeted groups only in 4 sites (Fig. 9), and only 6 sites had at least 20 patients vaccinated among the patients targeted 


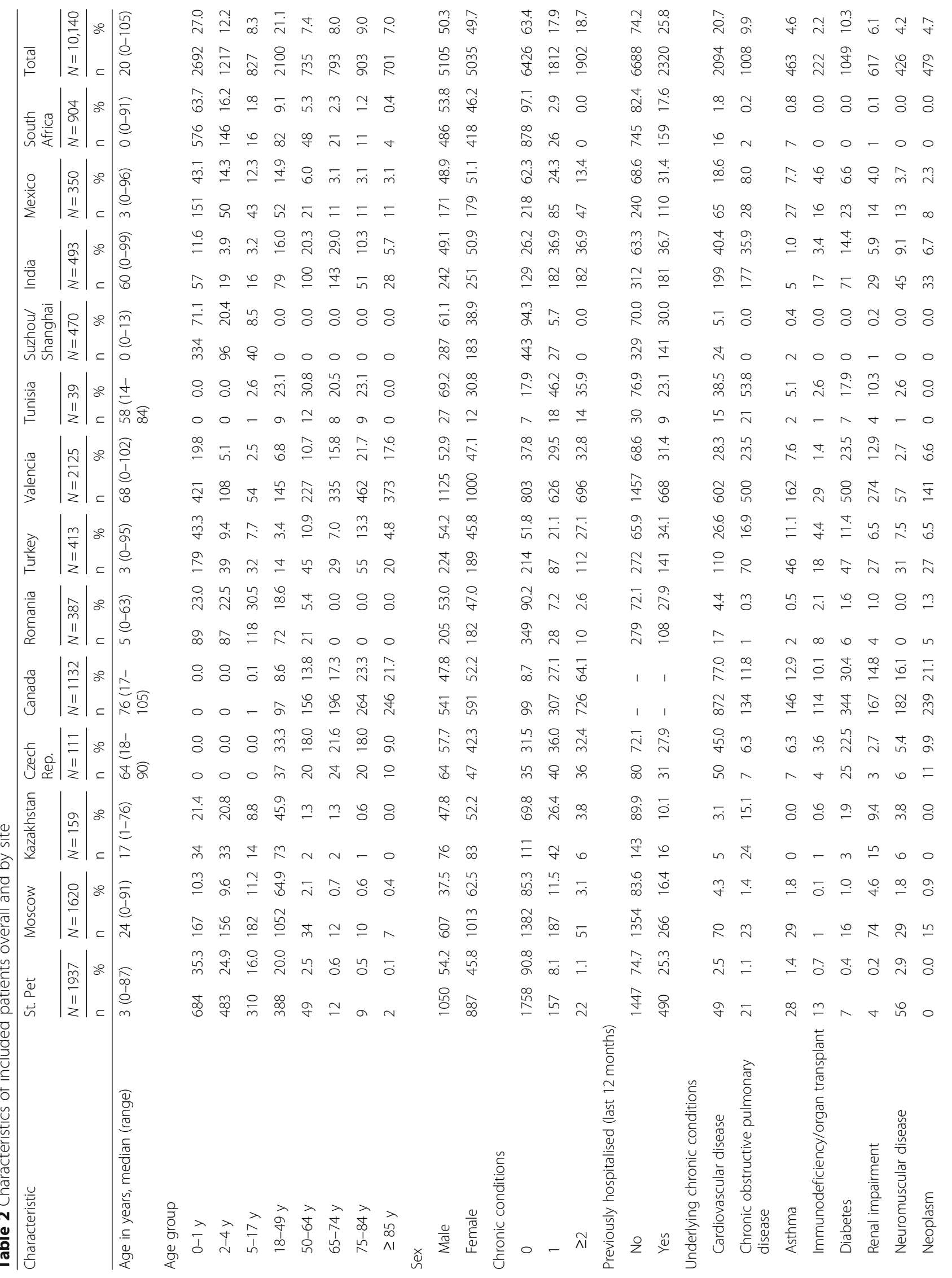




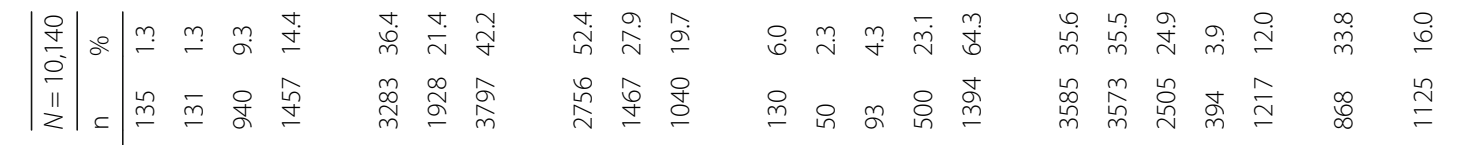
考

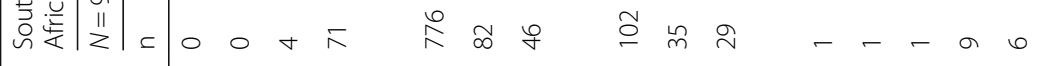

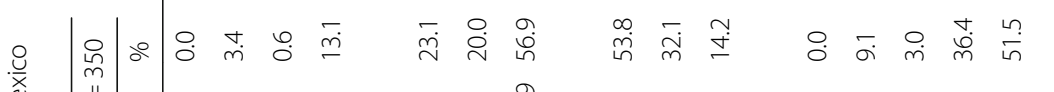

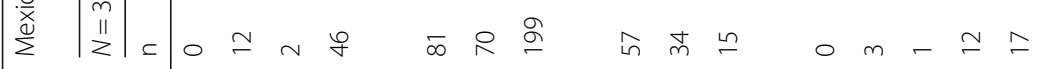

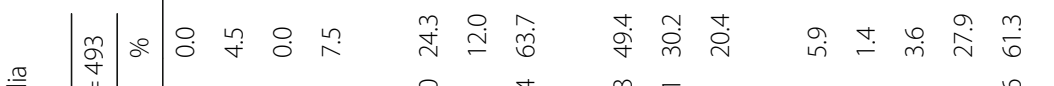

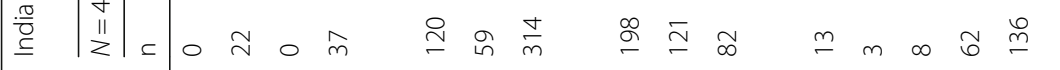

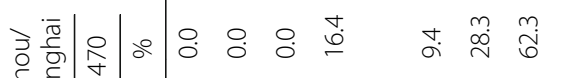

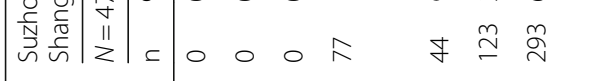

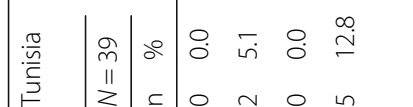

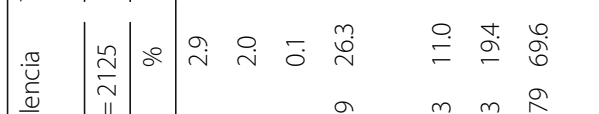

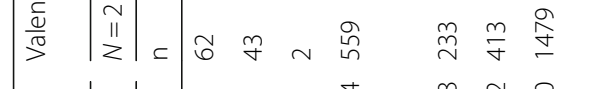

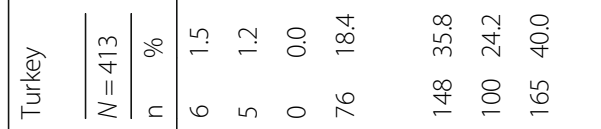

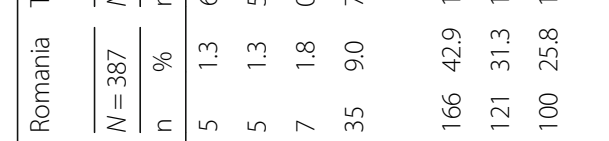

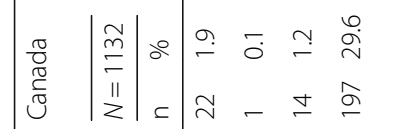
ஓ)

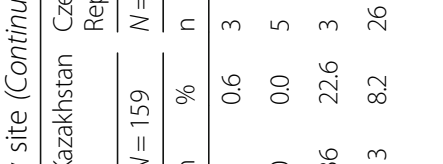

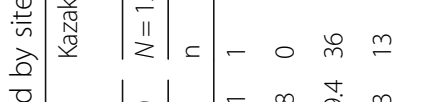

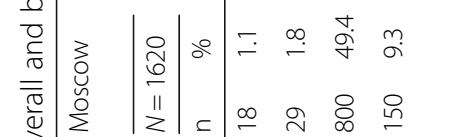

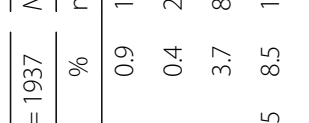
2. m $\dot{m}$ j

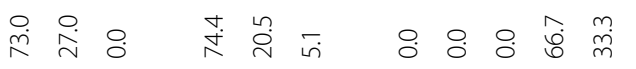

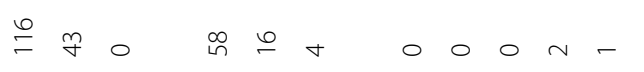
过守卞 $\quad$

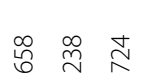

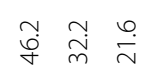
等

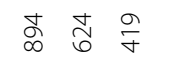

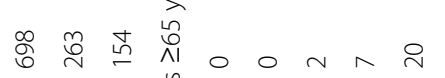

00000000

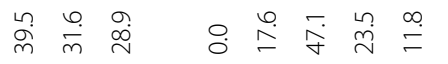

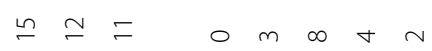

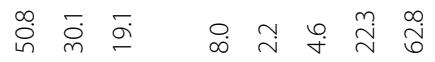

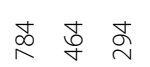

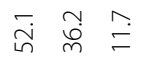

$\infty$ in

बं थู 芦

in $0 \stackrel{m}{m}$

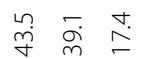

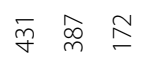

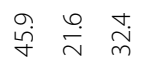

ปี

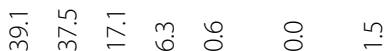

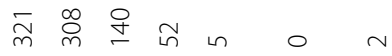
б 6)

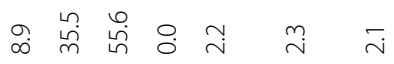
$\forall \stackrel{n}{\stackrel{\Delta}{\sim}} 0=$ n $\infty$

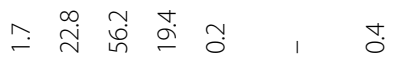

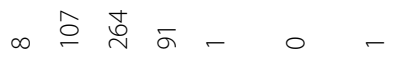

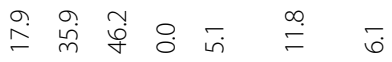

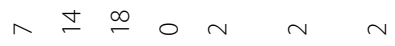

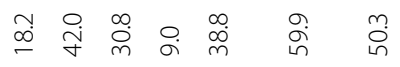

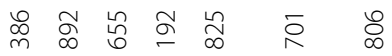

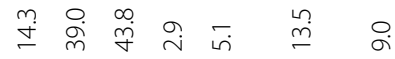

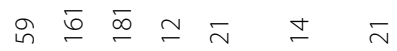

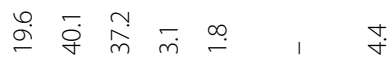

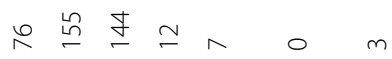

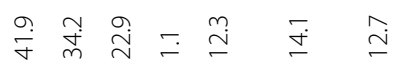
莳

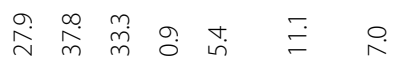
$\bar{m} \forall \hat{m}-006$ 吕

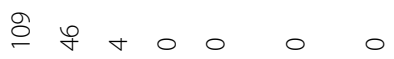
유ํ

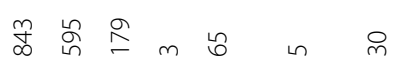
बे $\stackrel{\circ}{=} \stackrel{\infty}{\circ} \stackrel{人}{\sim} \circ \infty \sim \backsim$

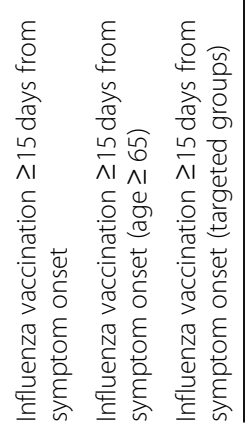




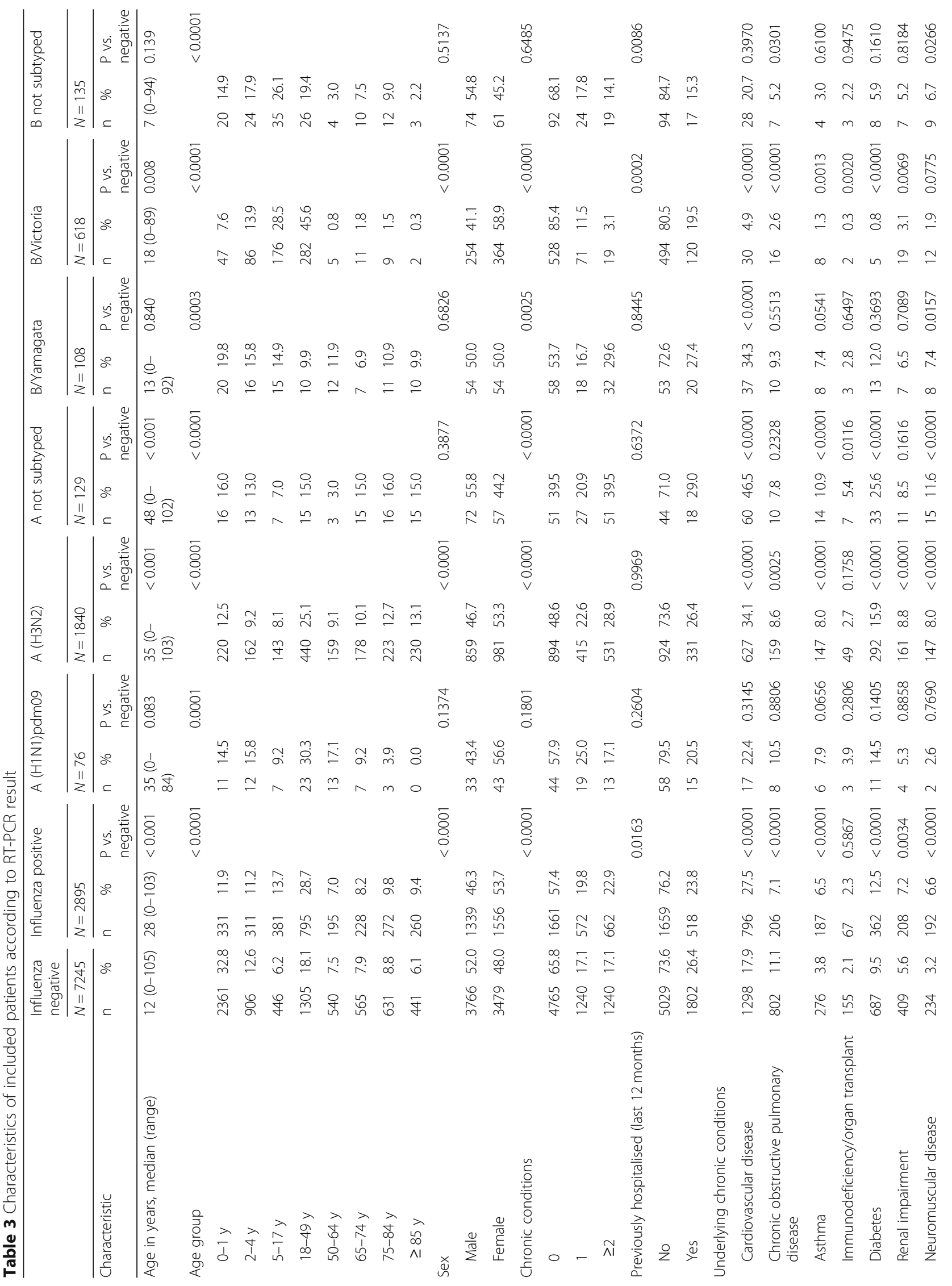




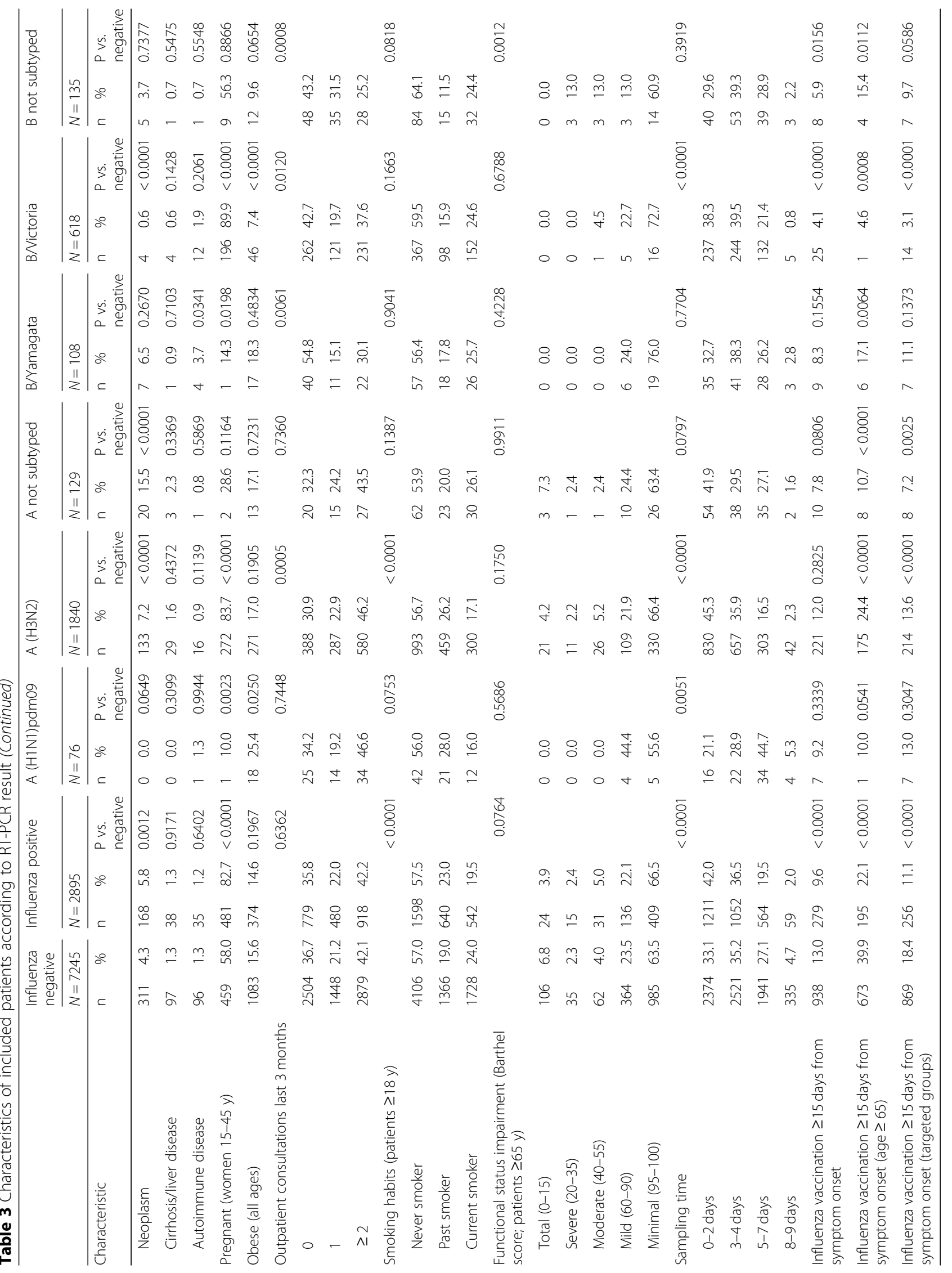


Table 4 Subject characteristics and risk of admission with influenza

\begin{tabular}{|c|c|c|c|c|c|c|c|c|}
\hline \multirow[b]{3}{*}{ Characteristic } & \multirow{3}{*}{$\begin{array}{l}\text { All admissions } \\
\mathrm{N}=10140 \\
\mathrm{~N}\end{array}$} & Influen & positive & \multicolumn{2}{|c|}{ Crude OR } & \multirow[t]{3}{*}{ Heterogeneity by strain $\left(1^{2}\right)$} & \multicolumn{2}{|l|}{$\mathrm{aOO}(*)$} \\
\hline & & \multicolumn{2}{|c|}{$N=2895$} & \multirow[b]{2}{*}{ Value } & \multirow[b]{2}{*}{$95 \% \mathrm{Cl}$} & & \multirow[b]{2}{*}{ Value } & \multirow[b]{2}{*}{$95 \% \mathrm{Cl}$} \\
\hline & & $\bar{N}$ & $\%$ & & & & & \\
\hline \multicolumn{9}{|l|}{ Age group } \\
\hline $0-1$ years & 2692 & 331 & 12.3 & 1.00 & - & $79.4 \%$ & 1.00 & - \\
\hline $2-4$ years & 1217 & 311 & 25.6 & 2.45 & $2.06-2.92$ & $75.6 \%$ & 0.86 & $0.67-1.09$ \\
\hline $5-17$ years & 827 & 381 & 46.1 & 6.09 & $5.03-7.38$ & $94.6 \%$ & 1.59 & $0.85-2.96$ \\
\hline $18-49$ years & 2100 & 795 & 37.9 & 4.35 & $3.73-5.06$ & $96.4 \%$ & 0.65 & $0.22-1.97$ \\
\hline 50-64 years & 735 & 195 & 26.5 & 2.58 & $2.10-3.15$ & $96.6 \%$ & 0.59 & $0.25-1.39$ \\
\hline $65-74$ years & 793 & 228 & 28.8 & 2.88 & $2.37-3.50$ & $95.3 \%$ & 0.61 & $0.31-1.22$ \\
\hline $75-84$ years & 903 & 272 & 30.1 & 3.07 & $2.55-3.71$ & $96.9 \%$ & 0.50 & $0.21-1.20$ \\
\hline$\geq 85$ years & 701 & 260 & 37.1 & 4.21 & $3.45-5.13$ & $98.4 \%$ & 0.49 & $0.19-1.28$ \\
\hline \multicolumn{9}{|l|}{ Sex } \\
\hline Male & 5105 & 1339 & $26,2 \%$ & 1.00 & & $54.0 \%$ & 1.00 & \\
\hline Female & 5035 & 1556 & $30,9 \%$ & 1.26 & $1.15-1.37$ & $46.5 \%$ & 0.84 & $0.74-0.95$ \\
\hline \multicolumn{9}{|l|}{ Smoking habits } \\
\hline Current smoker & 2270 & 542 & $23,9 \%$ & 1.00 & & $81.7 \%$ & 1.00 & \\
\hline Past smoker & 2006 & 640 & $31,9 \%$ & 1.49 & $1.30-1.71$ & $88.4 \%$ & 1.04 & $0.89-1.22$ \\
\hline Never smoker & 5704 & 1598 & $28,0 \%$ & 1.24 & $1.11-1.39$ & $34.0 \%$ & 1.09 & $0.93-1.28$ \\
\hline \multicolumn{9}{|l|}{ Consultations at the GP (last 3 months) } \\
\hline No & 3283 & 779 & $23,7 \%$ & 1.00 & & $95.0 \%$ & 1.00 & \\
\hline Yes & 5725 & 1398 & $24,4 \%$ & 1.04 & $0.94-1.15$ & $92.6 \%$ & 0.91 & $0.69-1.18$ \\
\hline \multicolumn{9}{|l|}{ Occupation / Social class } \\
\hline Qualified & 3810 & 1255 & $32,9 \%$ & 1.00 & & $97.1 \%$ & 1.00 & \\
\hline Skilled & 1376 & 355 & $25,8 \%$ & 0.71 & $0.62-0.81$ & $81.9 \%$ & 0.83 & $0.72-0.94$ \\
\hline Low or unskilled & 3411 & 591 & $17,3 \%$ & 0.43 & $0.38-0.48$ & $91.5 \%$ & 0.63 & $0.50-0.78$ \\
\hline \multicolumn{9}{|l|}{ Other risk factors } \\
\hline Comorbidity & 3714 & 1234 & $33,2 \%$ & 1.43 & $1.31-1.56$ & $98.7 \%$ & 0.90 & $0.63-1.30$ \\
\hline Cardiovascular disease & 2094 & 796 & $38,0 \%$ & 1.74 & $1.57-1.92$ & $98.7 \%$ & 1.01 & $0.72-1.40$ \\
\hline Chronic obstructive pulmonary disease & 1008 & 206 & $20,4 \%$ & 0.62 & $0.52-0.72$ & $92.5 \%$ & 0.66 & $0.45-0.98$ \\
\hline Asthma & 463 & 187 & $40,4 \%$ & 1.74 & $1.44-2.11$ & $94.3 \%$ & 1.31 & $0.96-1.77$ \\
\hline Immunodeficiency/organ transplant & 222 & 67 & $30,2 \%$ & 1.08 & $0.81-1.45$ & $85.2 \%$ & 0.57 & $0.28-1.17$ \\
\hline Diabetes & 1049 & 362 & $34,5 \%$ & 1.36 & $1.19-1.56$ & $98.1 \%$ & 1.19 & $1.03-1.37$ \\
\hline Chronic renal impairment & 617 & 208 & $33,7 \%$ & 1.29 & $1.09-1.54$ & $89.2 \%$ & 1.06 & $0.89-1.27$ \\
\hline Chronic neuromuscular disease & 426 & 192 & $45,1 \%$ & 2.13 & $1.75-2.59$ & $91.7 \%$ & 1.08 & $0.75-1.56$ \\
\hline Active neoplasm & 479 & 168 & $35,1 \%$ & 1.37 & $1.13-1.67$ & $96.8 \%$ & 0.63 & $0.42-0.95$ \\
\hline Chronic liver disease & 135 & 38 & $28,1 \%$ & 0.98 & $0.67-1.43$ & $38.8 \%$ & 1.09 & $0.79-1.50$ \\
\hline Autoimmune disease & 131 & 35 & $26,7 \%$ & 0.91 & $0.62-1.35$ & $23.8 \%$ & 1.14 & $0.84-1.56$ \\
\hline Obesity & 1457 & 374 & $25,7 \%$ & 0.92 & $0.81-1.04$ & $93.3 \%$ & 0.83 & $0.69-1.00$ \\
\hline Pregnancy & 942 & 483 & $51,3 \%$ & 2.96 & $2.58-3.40$ & $97.6 \%$ & 3.02 & $1.59-5.76$ \\
\hline \multicolumn{9}{|l|}{ Days from onset of symptoms to swabbing } \\
\hline $0-2$ days & 3585 & 1211 & $33,8 \%$ & 1.00 & & $92.8 \%$ & 1.00 & \\
\hline $3-4$ days & 3573 & 1052 & $29,4 \%$ & 0.82 & $0.74-0.90$ & $36.9 \%$ & 1.05 & $0.99-1.12$ \\
\hline 5-7 days & 2505 & 564 & $22,5 \%$ & 0.57 & $0.51-0.64$ & $83.4 \%$ & 0.82 & $0.64-1.07$ \\
\hline 8-9 days & 394 & 59 & $15,0 \%$ & 0.35 & $0.26-0.46$ & $65.2 \%$ & 0.60 & $0.47-0.77$ \\
\hline
\end{tabular}

\footnotetext{
${ }^{(*)}$ Adjusted Odds Ratios were obtained using the model described in the 'Methods' section (pg.6)
} 


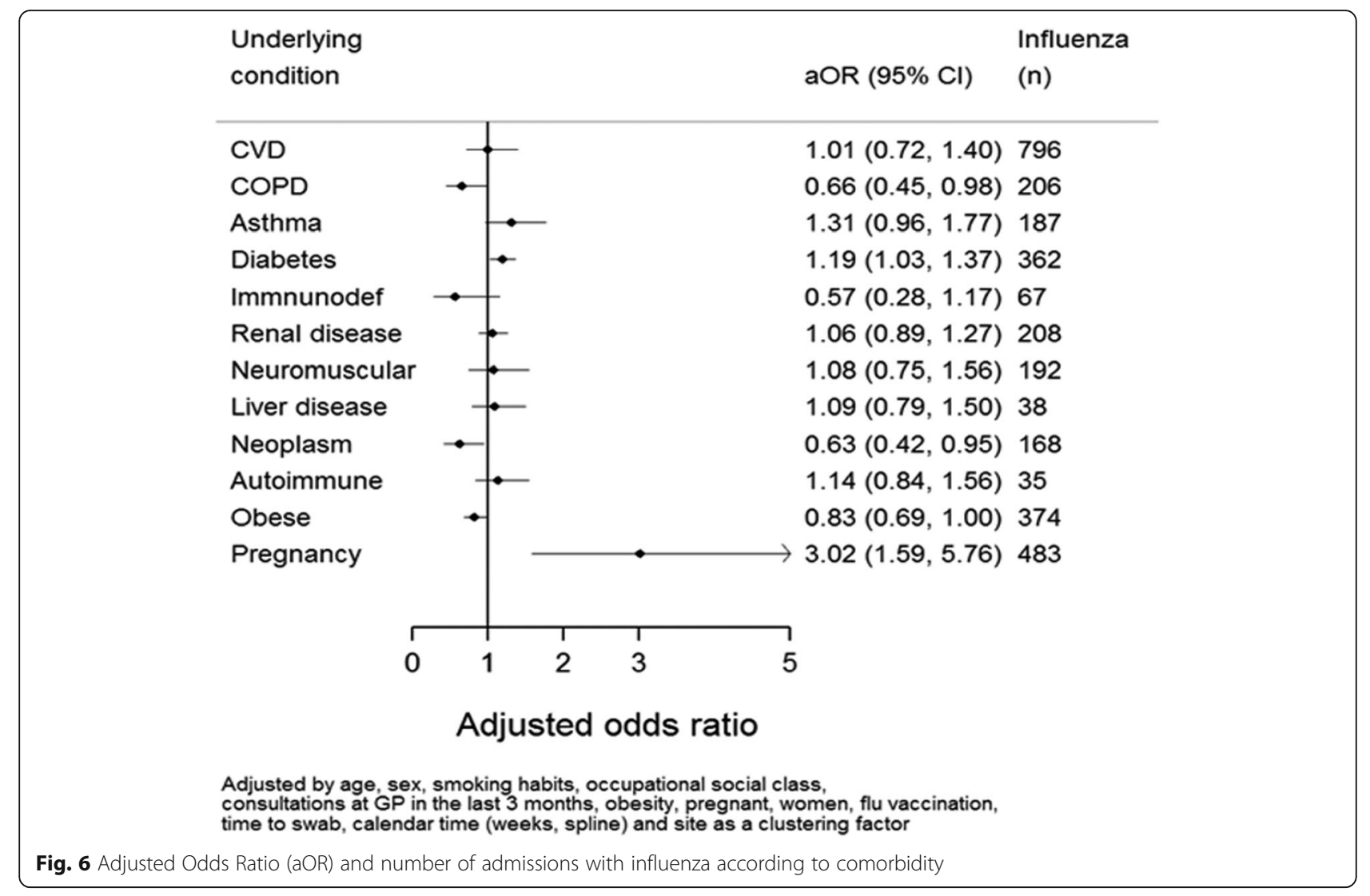

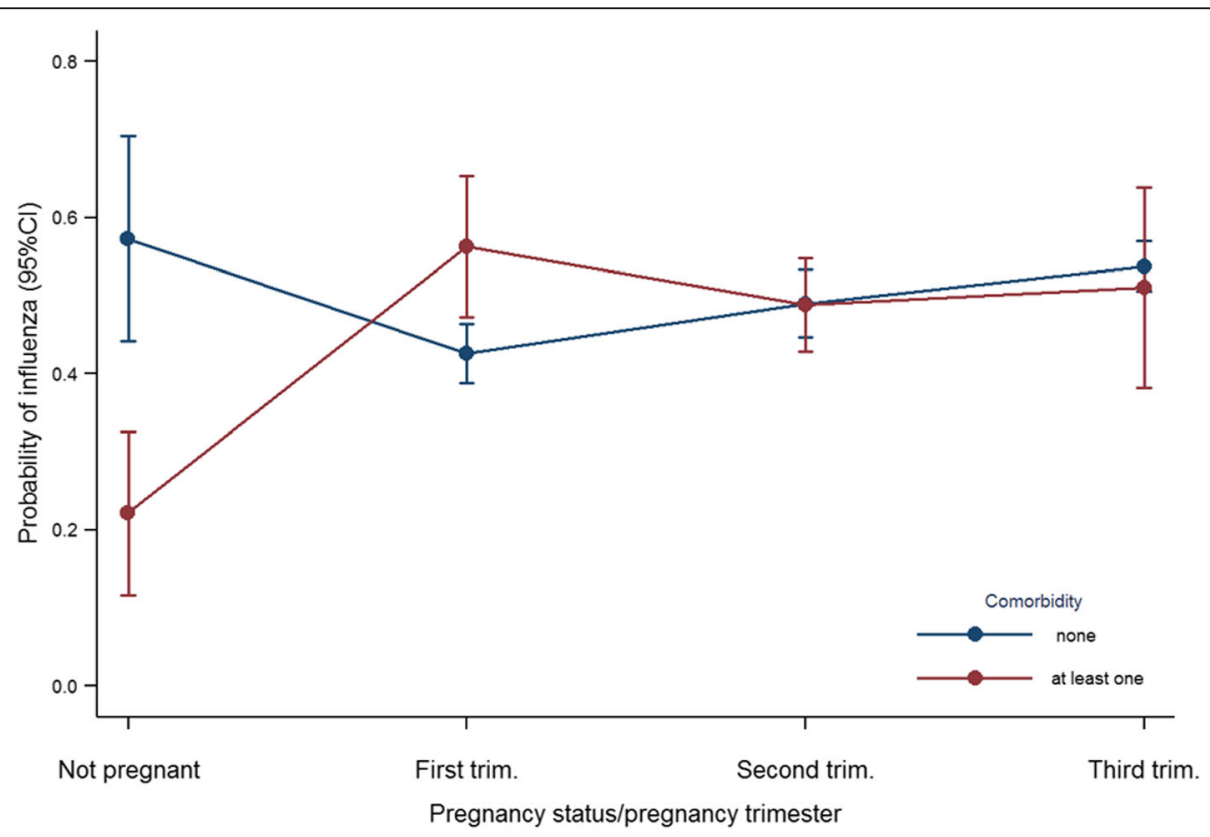

Fig. 7 Predicted probability of having an admission with influenza in pregnant and non-pregnant women by trimester 


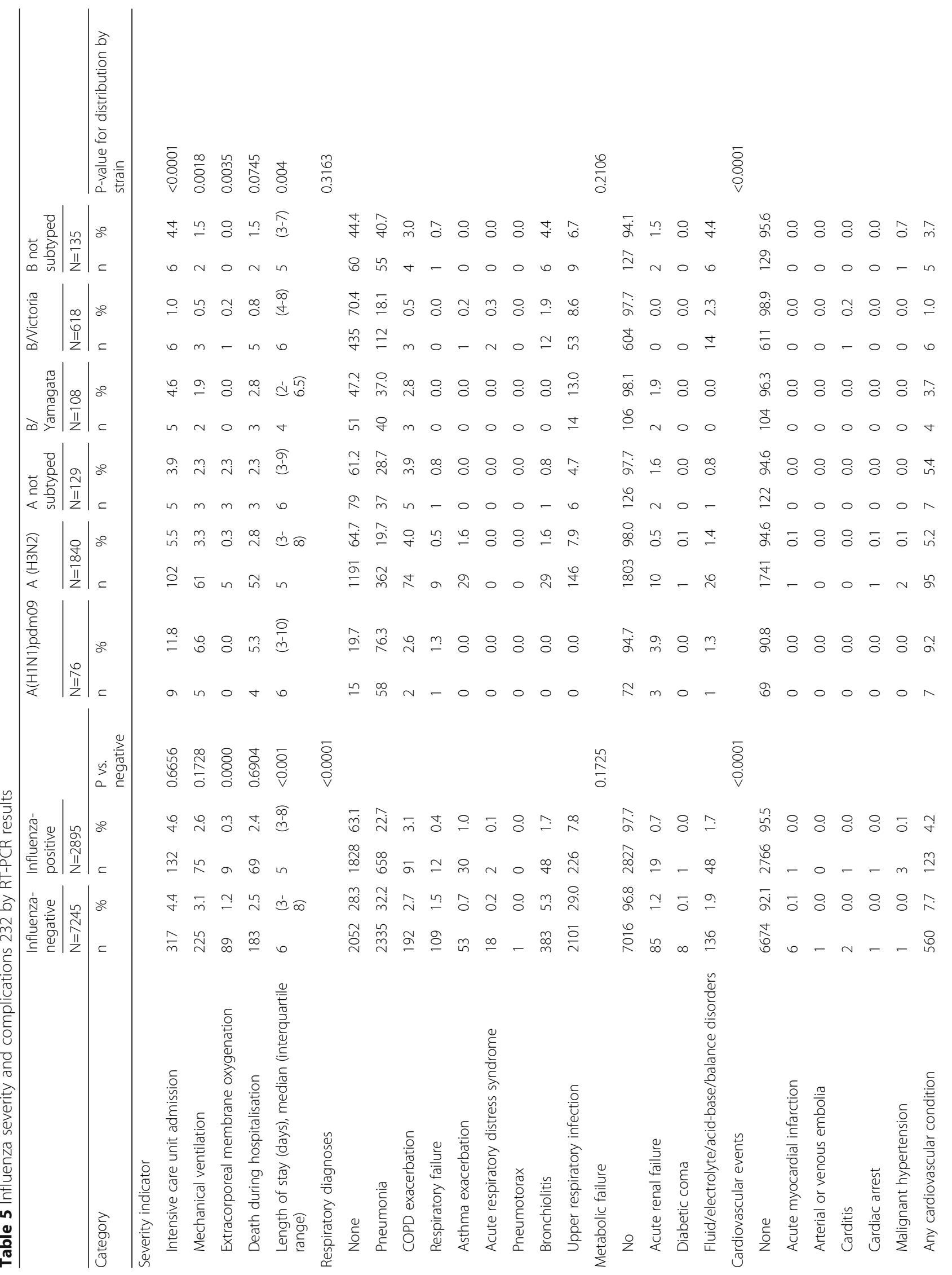


Baselga-Moreno et al. BMC Public Health (2019) 19:487

Page 15 of 23

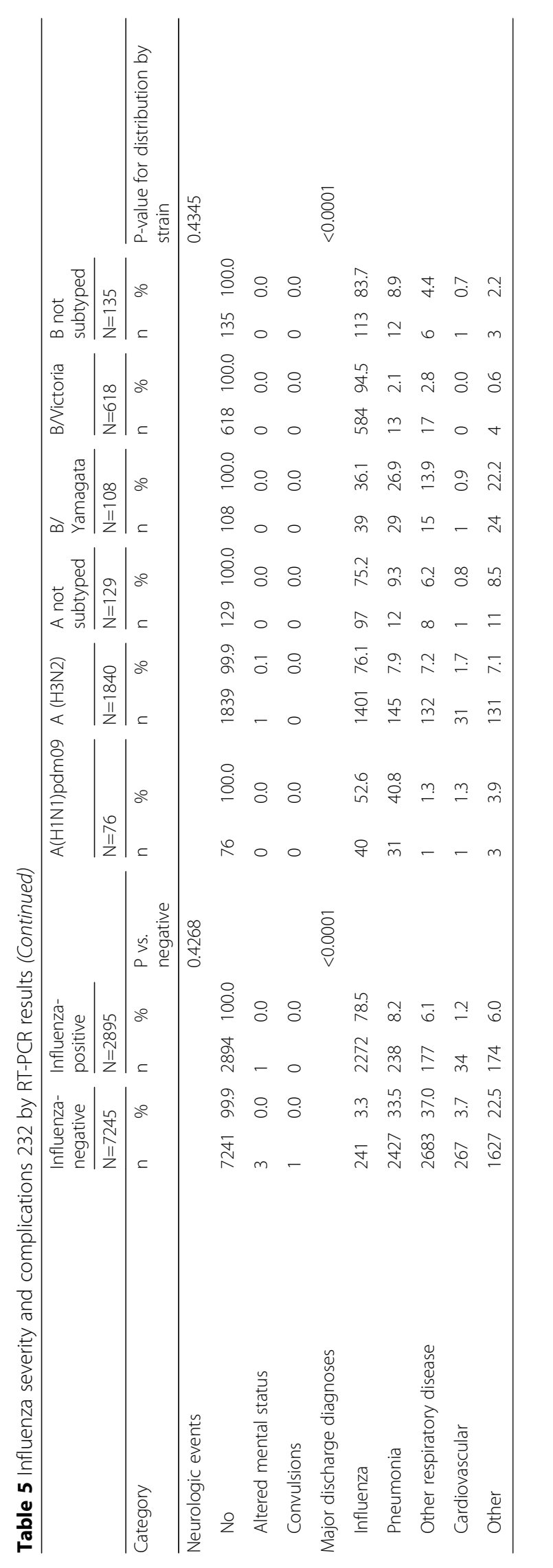




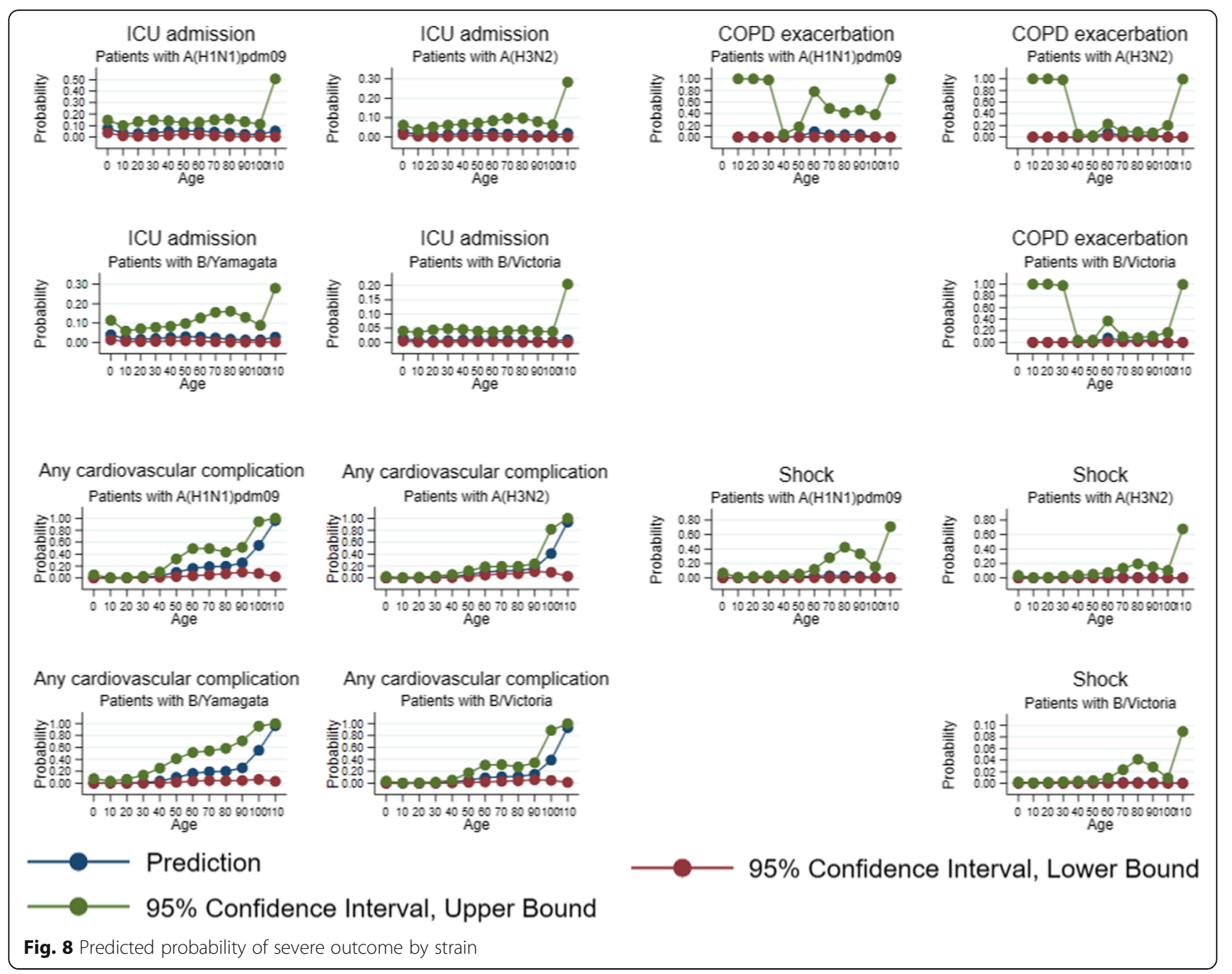

for vaccination. The IVE analysis was restricted to the sites with the highest vaccination coverage in targeted groups for vaccination having at least 20 patients vaccinated in these groups. These sites were Valencia, Canada, St. Petersburg, Mexico, Moscow and Turkey.

The IVE analysis, therefore, will be carried out in these six sites and globally. Vaccination coverage in pregnant women was $0 \%$ in Kazakhstan among the included patients, and in Moscow, only 1.3\% (10 out of 800 ) of the admitted pregnant women received the vaccine at least 15 days before symptoms onset, therefore, adjusted IVE could not be estimated for pregnant women.

Vaccination coverage was higher in patients older than 65 years and in patients with two or more comorbidities. Among immunized women 15 to 45 years old, 19 of 47 were pregnant $(40.4 \%)$, and among all vaccinated patients, $26.7 \%$ were obese.

Of the subjects vaccinated, $78.0 \%$ were also vaccinated in season $2015-2016$ and $67.2 \%$ were vaccinated in season $2014-2015$. However, $8.0 \%$ of the unvaccinated patients in the current season were vaccinated in the season 2015-2016, and 6.6\% in the season 2014-2015 (Table 6).

\section{IVE estimates for included patients}

In the selected sites for IVE estimates, vaccination coverage was $11.7 \%$ among the influenza positives and $22.2 \%$ among the influenza negatives. The overall IVE was 27.24\% (95\% CI 15.62 to $37.27 \%$ ) in targeted groups for vaccination. Table 7 shows IVE for different strains, Fig. 10 by study country.

IVE was statistically significant for all strains except for $\mathrm{A}(\mathrm{H} 1 \mathrm{~N} 1) \mathrm{pdm} 09$ due to the limited sample size, and the point estimate was higher for both influenza B lineages, even using the trivalent vaccine (Fig. 11). Heterogeneity among influenza types/subtypes was relevant $\left(\mathrm{I}^{2}=57.4 \%\right)$.

This season IVE estimate was higher in patients 85 years old or older (51.17\% [95\% CI: 35.13 to 63.24]). IVE was also high and statistically significant for patients 2 to 4 years old (49.37\% [95\% CI: 21.60 to 67.30]) (Fig. 12). Heterogeneity among the different age groups was relevant $\left(\mathrm{I}^{2}=69 \%\right)$. 


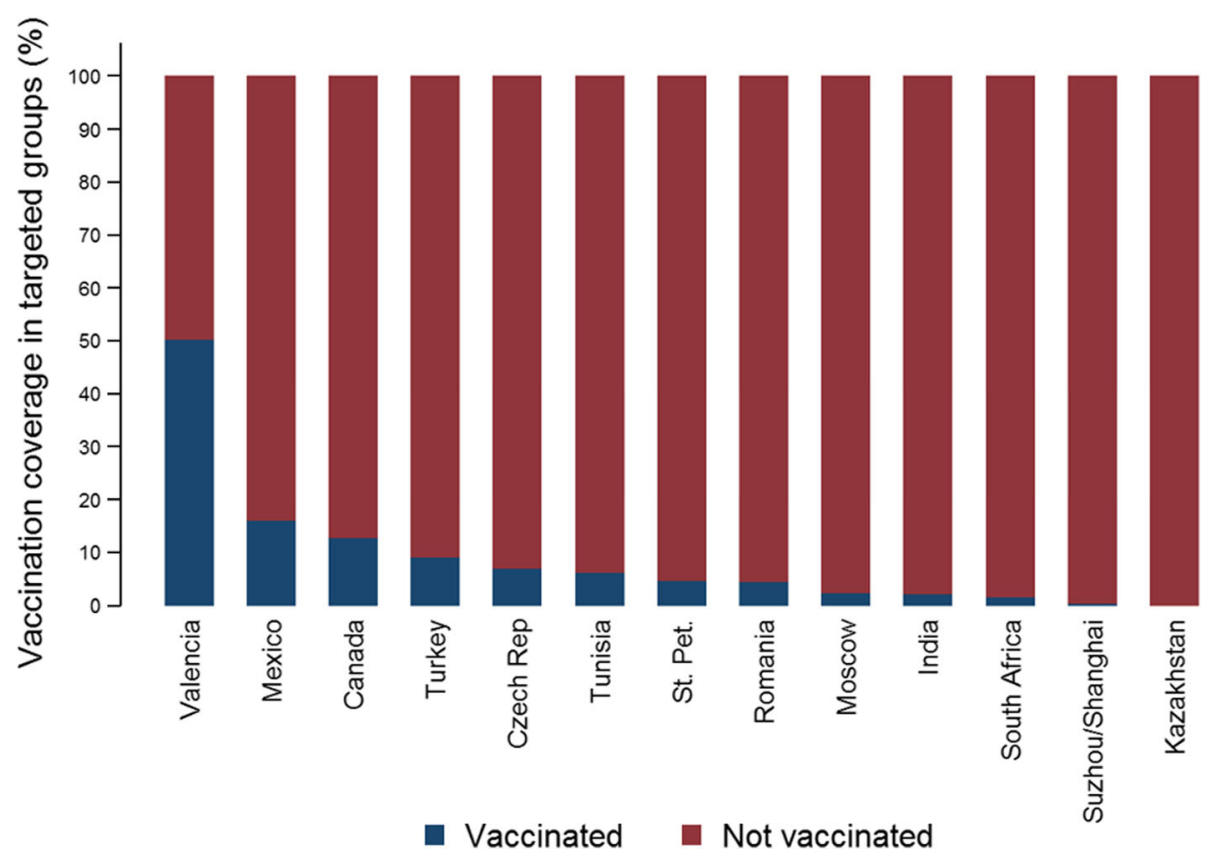

Fig. 9 Vaccination coverage in targeted groups by site

\section{Discussion}

The GIHSN included sites from the two hemispheres in the 2016/17 season. However, Ivory Coast and Peru were not included in the epidemiology study or in the IVE study due to the low influenza cases detected. This season was characterized by a predominance in the circulation of $\mathrm{A}(\mathrm{H} 3 \mathrm{~N} 2)$ virus, and a second wave of B/Victoria. However, A(H1N1)pdm09 was predominant in Mexico. B/Yamagata-strain, which was not included in the vaccine, also circulated in some areas.

Influenza $\mathrm{A}(\mathrm{H} 1 \mathrm{~N} 1) \mathrm{pdm} 09$ was mainly found in Mexico. A low vaccination coverage was seen in most of the GIHSN sites.

The GIHSN represents an opportunity to analyse the epidemiology of hospitalized influenza cases, and an assessment of the vaccine effectiveness worldwide. However, there are some limitations that should be mentioned:

- Although the same protocol was developed, the adaptation to different countries or sites produced some heterogeneity in the results, as previously reported in the network [3].

- In general vaccination coverage was low in most sites, even among high risk groups.

- Other factors as number of cases per site, and variability in the vaccination coverage, increased the heterogeneity in the reporting and analysis.
All of these limitations contributed to the complexity of the interpretation of the results.

In the northern hemisphere, the season differed by latitude [14], and this may have implications in the calendar of the vaccination campaigns.

Patients tested for influenza 8 to 9 days after symptoms onset had a higher proportion of samples negative for influenza than patients tested within the first 7 days after symptoms onset, as that viral load decreases with increasing time since infection, [15]. However, there were a few cases in our study as we collected all cases whose admission was in the 7 days after ILI symptoms started, and any delay in approaching the patient could result in a late swabbing.

Among inpatients with COPD, there was not a higher risk of testing for influenza. As all the cases were hospitalized, this result cannot be interpreted as COPD not being a risk factor for influenza hospitalization, as any other respiratory infection may decompensate the respiratory condition and force an admission. Besides vaccination coverage is higher in subjects with chronic conditions [16] and therefore, protection from the vaccine may also impact on our finding.

The risk of testing positive for influenza in diabetic patients was slightly higher than non-diabetic patients, as it also happened in previous seasons [3, 4]. Pregnancy also increased the probability of having influenza in women, particularly if they had at least one comorbidity in the first trimester. 
Table $\mathbf{6}$ Characteristics of patients included in the primary analysis by vaccination status

\begin{tabular}{|c|c|c|c|c|c|c|}
\hline \multirow[t]{2}{*}{ Risk variables } & \multirow[b]{2}{*}{ Category } & \multicolumn{2}{|c|}{ Unvaccinated } & \multicolumn{2}{|c|}{ Vaccinated } & \multirow[t]{2}{*}{$P$ value } \\
\hline & & $n$ & $\%$ & $\mathrm{n}$ & $\%$ & \\
\hline \multirow[t]{2}{*}{ Number of patients, n (\%) } & Controls & 6307 & 70.7 & 938 & 77.1 & \multirow[t]{2}{*}{$<0.0001$} \\
\hline & Cases & 2616 & 29.3 & 279 & 22.9 & \\
\hline Age $(y)$ & Median (range) & \multicolumn{2}{|c|}{$11.4(0-105.3)$} & \multicolumn{2}{|c|}{$76.5(0.6-102.8)$} & $<0.0001$ \\
\hline \multirow[t]{9}{*}{ Age group, n (\%) ${ }^{(2)}$} & $0-5$ months & 1254 & $14.3 \%$ & 0 & $0.0 \%$ & \multirow[t]{9}{*}{$<0.0001$} \\
\hline & 6-11 months & 643 & $7.3 \%$ & 13 & $1.1 \%$ & \\
\hline & $1-4$ yrs & 1948 & $22.2 \%$ & 51 & $4.3 \%$ & \\
\hline & $5-17 y r s$ & 760 & $8.7 \%$ & 67 & $5.6 \%$ & \\
\hline & $18-49 y r s$ & 1988 & $22.7 \%$ & 112 & $9.4 \%$ & \\
\hline & $50-64$ yrs & 628 & $7.2 \%$ & 106 & $8.9 \%$ & \\
\hline & $65-74$ yrs & 583 & $6.6 \%$ & 210 & $17.6 \%$ & \\
\hline & $75-84$ yrs & 566 & $6.5 \%$ & 337 & $28.2 \%$ & \\
\hline & $\geq 85 y$ & 403 & $4.6 \%$ & 299 & $25.0 \%$ & \\
\hline \multirow[t]{2}{*}{ Sex, n (\%) } & Male & 4462 & $50.0 \%$ & 643 & $52.8 \%$ & \multirow[t]{2}{*}{0.0641} \\
\hline & Female & 4461 & $50.0 \%$ & 574 & $47.2 \%$ & \\
\hline \multirow[t]{3}{*}{ Comorbidities, n (\%) } & None & 6123 & $68.6 \%$ & 303 & $24.9 \%$ & \multirow[t]{3}{*}{$<0.0001$} \\
\hline & 1 & 1457 & $16.3 \%$ & 355 & $29.2 \%$ & \\
\hline & $>1$ & 1343 & $15.1 \%$ & 559 & $45.9 \%$ & \\
\hline Pregnant, n (\%) & - & 921 & $69.5 \%$ & 19 & $40.4 \%$ & $<0.0001$ \\
\hline Obesity, n (\%) & - & 1148 & $13.8 \%$ & 309 & $26.7 \%$ & $<0.0001$ \\
\hline Previous hospitalisation within 12 months, n (\%) & - & 1914 & $24.1 \%$ & 406 & $37.7 \%$ & $<0.0001$ \\
\hline \multirow[t]{3}{*}{ GP visit within 3 months, $\mathrm{n}(\%)$} & None & 3074 & $38.8 \%$ & 209 & $19.4 \%$ & \multirow[t]{3}{*}{$<0.0001$} \\
\hline & 1 & 1740 & $21.9 \%$ & 188 & $17.4 \%$ & \\
\hline & $>1$ & 3116 & $39.3 \%$ & 681 & $63.2 \%$ & \\
\hline \multirow[t]{3}{*}{ Smoking, n (\%) } & Current & 2112 & $24.1 \%$ & 158 & $13.0 \%$ & \multirow[t]{3}{*}{$<0.0001$} \\
\hline & Past & 1618 & $18.5 \%$ & 388 & $32.0 \%$ & \\
\hline & Never & 5037 & $57.5 \%$ & 667 & $55.0 \%$ & \\
\hline \multirow[t]{5}{*}{ Functional impairment in $\geq 65 \mathrm{y}, \mathrm{n}(\%)$} & None or minimal & 72 & $5.4 \%$ & 58 & $7.0 \%$ & \multirow[t]{5}{*}{0.4086} \\
\hline & Mild & 32 & $2.4 \%$ & 18 & $2.2 \%$ & \\
\hline & Moderate & 52 & $3.9 \%$ & 41 & $4.9 \%$ & \\
\hline & Severe & 309 & $23.1 \%$ & 191 & $23.0 \%$ & \\
\hline & Total & 871 & $65.2 \%$ & 523 & $62.9 \%$ & \\
\hline Sampling interval (days) & Median (range) & $3(0-9)$ & & $4(0-9)$ & & $<0.0001$ \\
\hline \multirow[t]{3}{*}{ Sampling interval, n (\%) } & $\leq 4$ days & 6377 & $72.1 \%$ & 781 & $64.2 \%$ & \multirow[t]{3}{*}{$<0.0001$} \\
\hline & 5-7 days & 2148 & $24.3 \%$ & 357 & $29.3 \%$ & \\
\hline & 8-9 days & 315 & $3.6 \%$ & 79 & $6.5 \%$ & \\
\hline Site, n (\%) & St. Pet & 1851 & $20.7 \%$ & 86 & $7.1 \%$ & $<0.0001$ \\
\hline & Moscow & 1555 & $17.4 \%$ & 65 & $5.3 \%$ & \\
\hline & Kazakhstan & 159 & $1.8 \%$ & 0 & $0.0 \%$ & \\
\hline & Czech Republic & 105 & $1.2 \%$ & 6 & $0.5 \%$ & \\
\hline & Canada & 993 & $11.1 \%$ & 139 & $11.4 \%$ & \\
\hline & Romania & 380 & $4.3 \%$ & 7 & $0.6 \%$ & \\
\hline & Turkey & 392 & $4.4 \%$ & 21 & $1.7 \%$ & \\
\hline & Valencia & 1300 & $14.6 \%$ & 825 & $67.8 \%$ & \\
\hline
\end{tabular}


Table $\mathbf{6}$ Characteristics of patients included in the primary analysis by vaccination status (Continued)

\begin{tabular}{|c|c|c|c|c|c|c|}
\hline \multirow[t]{2}{*}{ Risk variables } & \multirow[b]{2}{*}{ Category } & \multicolumn{2}{|c|}{ Unvaccinated } & \multicolumn{2}{|c|}{ Vaccinated } & \multirow[t]{2}{*}{$P$ value } \\
\hline & & $n$ & $\%$ & $n$ & $\%$ & \\
\hline & Tunisia & 37 & $0.4 \%$ & 2 & $0.2 \%$ & \\
\hline & Suzhou/Shanghai & 469 & $5.3 \%$ & 1 & $0.1 \%$ & \\
\hline & India & 482 & $5.4 \%$ & 11 & $0.9 \%$ & \\
\hline & Mexico & 301 & $3.4 \%$ & 49 & $4.0 \%$ & \\
\hline & South Africa & 899 & $10.1 \%$ & 5 & $0.4 \%$ & \\
\hline \multirow[t]{2}{*}{ Vaccinated, n (\%) } & In 2015-2016 & 718 & $8.0 \%$ & 949 & $78.0 \%$ & $<0.0001$ \\
\hline & In 2014-2015 & 589 & $6.6 \%$ & 818 & $67.2 \%$ & $<0.0001$ \\
\hline
\end{tabular}

Table 7 IVE for all cases and for targeted groups only by age and strain

\begin{tabular}{|c|c|c|c|c|c|c|c|c|}
\hline \multirow{2}{*}{ Population } & \multirow[b]{2}{*}{ Strain } & \multirow[b]{2}{*}{ Age } & \multicolumn{2}{|c|}{ Influenza-positive } & \multicolumn{2}{|c|}{ Influenza-negative } & \multicolumn{2}{|l|}{ Adjusted IVE ${ }^{(*)}$} \\
\hline & & & $\overline{\text { Total }}$ & Vaccinated & Total & Vaccinated & $\begin{array}{l}\text { Percent } \\
(95 \% \mathrm{Cl})\end{array}$ & P-value \\
\hline \multirow[t]{15}{*}{ Overall } & \multirow[t]{3}{*}{ Any } & Any & 2895 & 279 & 7245 & 938 & $27(15,38)$ & \multirow{3}{*}{0.804} \\
\hline & & $<65 y$ & 2013 & 84 & 5558 & 265 & $27(-1,48)$ & \\
\hline & & $\geq 65 y$ & 882 & 195 & 1687 & 673 & $25(3,43)$ & \\
\hline & \multirow[t]{3}{*}{ A (H1N1) pdm09 } & Any & 76 & 7 & 7245 & 938 & $39(-68,78)$ & \multirow{3}{*}{0.346} \\
\hline & & $<65 y$ & 66 & 6 & 5558 & 265 & $2(-138,60)$ & \\
\hline & & $\geq 65 y$ & 10 & 1 & 1687 & 673 & $99(1,100)$ & \\
\hline & \multirow[t]{3}{*}{$\mathrm{A}(\mathrm{H} 3 \mathrm{~N} 2)$} & Any & 1840 & 221 & 7245 & 938 & $25(13,35)$ & \multirow{3}{*}{0.703} \\
\hline & & $<65 y$ & 1124 & 46 & 5558 & 265 & $31(1,51)$ & \\
\hline & & $\geq 65 y$ & 716 & 175 & 1687 & 673 & $19(-10,40)$ & \\
\hline & \multirow[t]{3}{*}{ B/Yamagata } & Any & 108 & 9 & 7245 & 938 & $41(-110,84)$ & \multirow{3}{*}{0.203} \\
\hline & & $<65 y$ & 73 & 3 & 5558 & 265 & $7(-178,69)$ & \\
\hline & & $\geq 65 y$ & 35 & 6 & 1687 & 673 & $73(-38,95)$ & \\
\hline & \multirow[t]{3}{*}{ B/Nictoria } & Any & 618 & 25 & 7245 & 938 & $43(-15,71)$ & \multirow{3}{*}{0.191} \\
\hline & & $<65 y$ & 596 & 24 & 5558 & 265 & $27(-14,54)$ & \\
\hline & & $\geq 65 y$ & 22 & 1 & 1687 & 673 & $89(40,98)$ & \\
\hline \multirow[t]{15}{*}{ Targeted groups only } & \multirow[t]{3}{*}{ Any } & Any & 2314 & 256 & 4723 & 869 & $27(16,37)$ & \multirow{3}{*}{0.657} \\
\hline & & $<65 y$ & 1432 & 61 & 3036 & 196 & $37(0,47)$ & \\
\hline & & $\geq 65 y$ & 882 & 195 & 1687 & 673 & $25(3,43)$ & \\
\hline & \multirow[t]{3}{*}{ A (H1N1) pdm09 } & Any & 54 & 7 & 4723 & 869 & $18(-142,72)$ & \multirow{3}{*}{0.423} \\
\hline & & $<65 y$ & 44 & 6 & 3036 & 196 & $-62(-303,35)$ & \\
\hline & & $\geq 65 y$ & 10 & 1 & 1687 & 673 & $99(1,100)$ & \\
\hline & \multirow[t]{3}{*}{$\mathrm{A}(\mathrm{H} 3 \mathrm{~N} 2)$} & Any & 1572 & 214 & 4723 & 869 & $23(9,34)$ & \multirow{3}{*}{0.485} \\
\hline & & $<65 y$ & 856 & 39 & 3036 & 196 & $27(-7,50)$ & \\
\hline & & $\geq 65 y$ & 716 & 175 & 1687 & 673 & $19(-10,40)$ & \\
\hline & \multirow[t]{3}{*}{ B/Yamagata } & Any & 63 & 7 & 4723 & 869 & $72(8,92)$ & \multirow{3}{*}{0.037} \\
\hline & & $<65 y$ & 28 & 1 & 3036 & 196 & $65(-35,91)$ & \\
\hline & & $\geq 65 y$ & 35 & 6 & 1687 & 673 & $73(-38,95)$ & \\
\hline & \multirow[t]{3}{*}{ B/Nictoria } & Any & 449 & 14 & 4723 & 869 & $66(3,80)$ & \multirow{3}{*}{0.262} \\
\hline & & $<65 y$ & 427 & 13 & 3036 & 196 & $41(10,62)$ & \\
\hline & & $\geq 65 y$ & 22 & 1 & 1687 & 673 & $89(40,98)$ & \\
\hline
\end{tabular}




\section{Adjusted}

Influenza Vaccine

Site

Effectiveness $(95 \% \mathrm{Cl})$

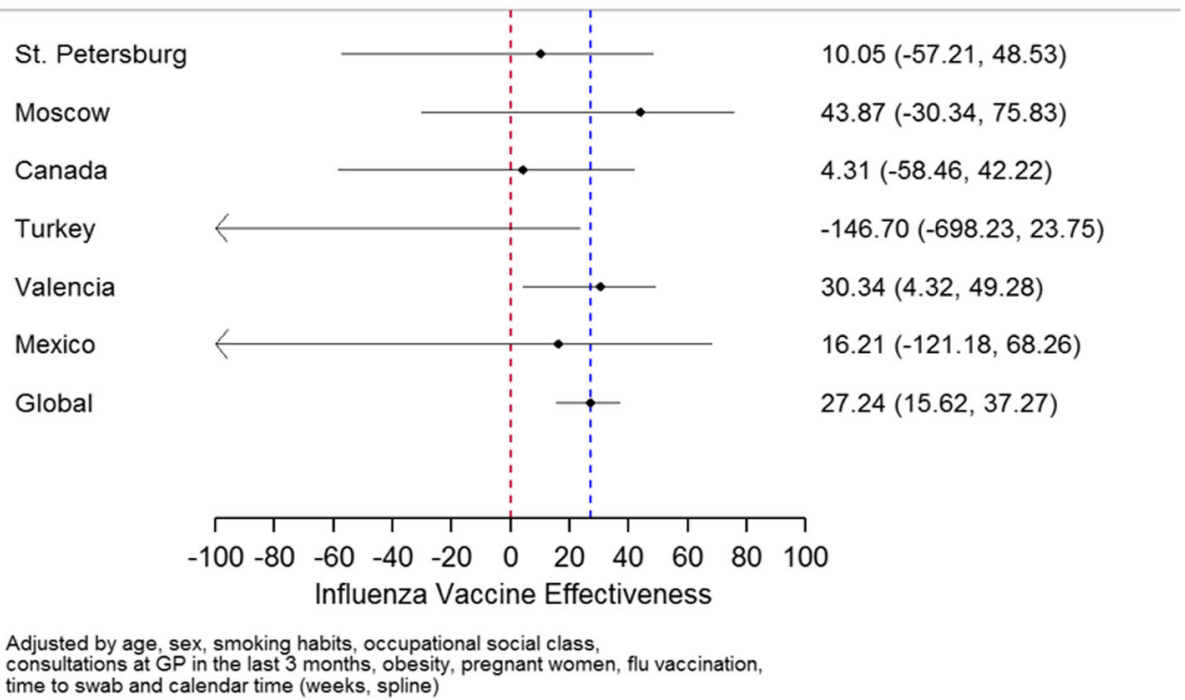

Fig. 10 Adjusted Influenza Vaccine Effectiveness by site

Adjusted

Influenza Vaccine

Strain

Effectiveness $(95 \% \mathrm{Cl})$

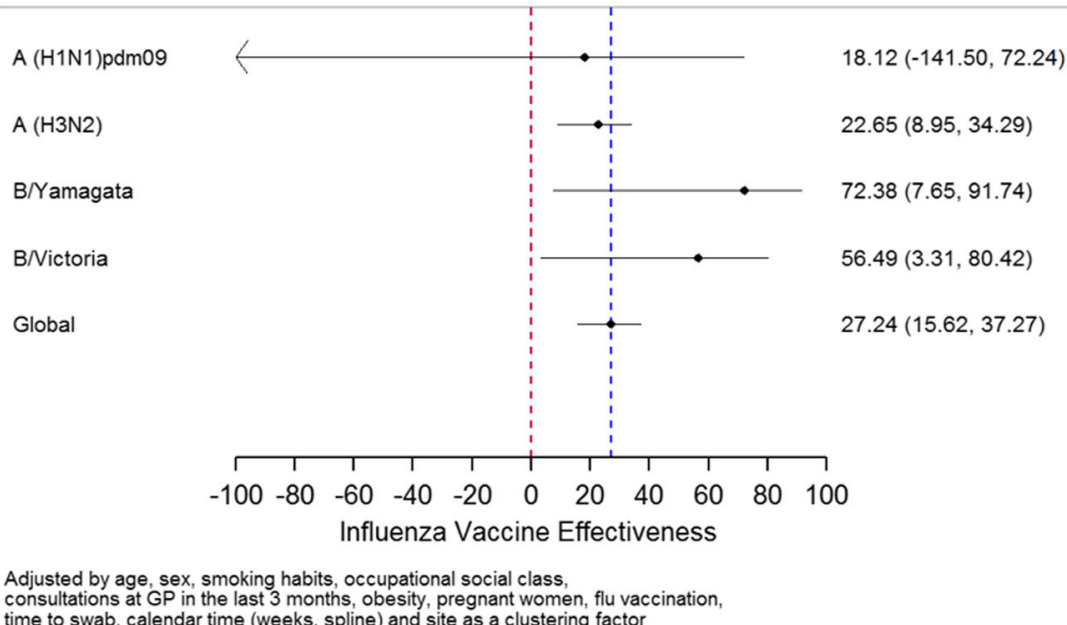

consultations at $G P$ in the last 3 months, obesity, pregnant women, flu vaccination,

Fig. 11 Adjusted Influenza Vaccine Effectiveness by strain 


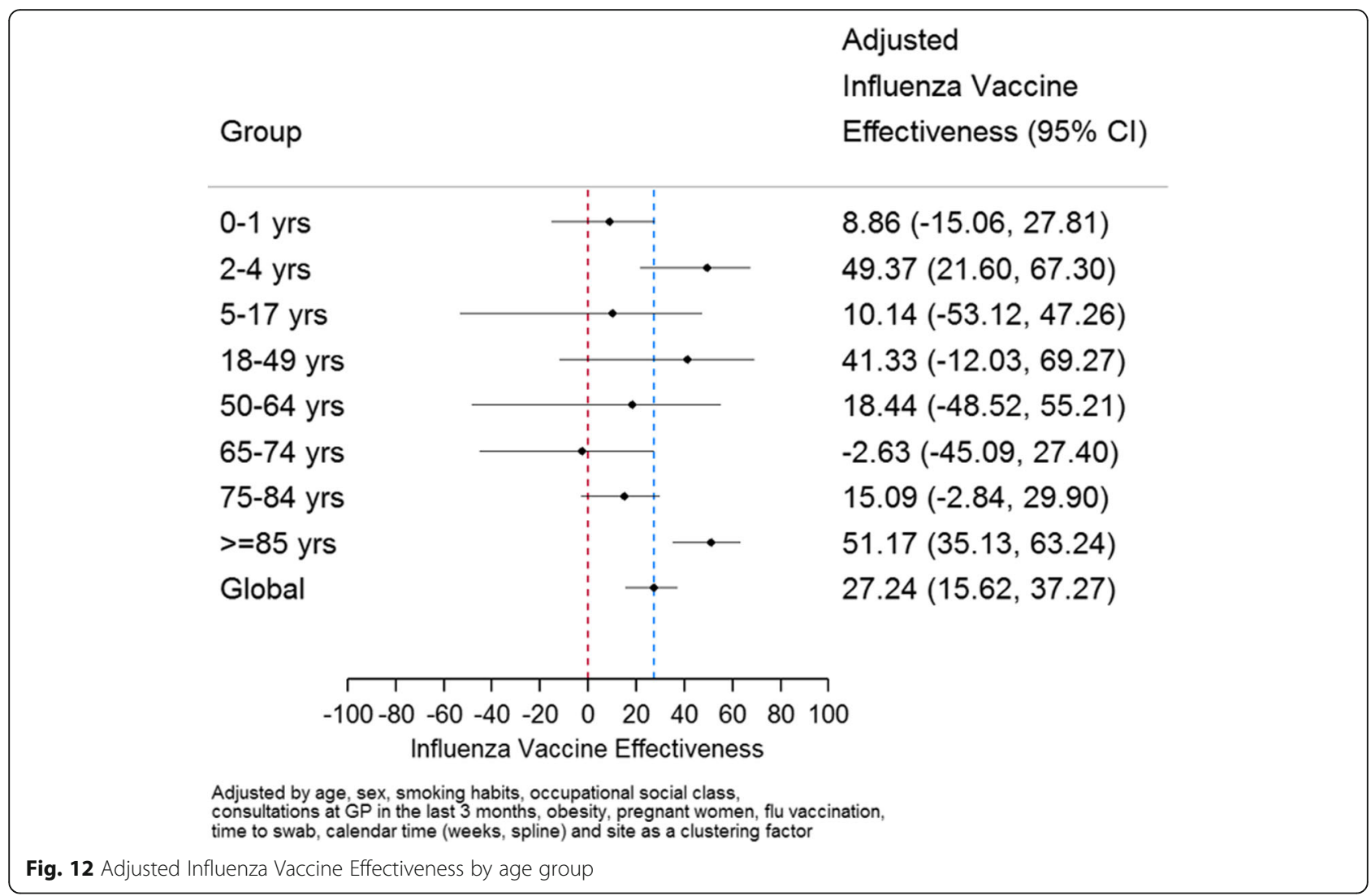

Despite differences in the characteristics of the included patients relative to the age or pregnancy status, heterogeneity in the IVE analysis among the 6 sites with the highest numbers of vaccinated patients was low. Point estimates of the overall IVE from a two-step pooling was $27.2 \%$ (95\% CI: 15.62 to 37.27 ) in hospitalized, which is higher than that reported in Europe for hospitalised patients [17], that ranged from 2.4 to $7.9 \%$, depending on the age group, and lower to that estimated by the US CDC, which was $40 \%$ (95\% CI: 32 to 46) [18].

Pooled Influenza vaccine effectiveness showed protection against all influenza virus that circulated, although for $\mathrm{A}(\mathrm{H} 1 \mathrm{~N} 1) \mathrm{pdm} 09$ did not reach statistical significance, as the circulation of the virus was low except in Mexico. There was a significant effectiveness against both B lineages, even though most of the vaccines used were trivalent, i.e. only contained the $\mathrm{B} /$ Victoria linage, following recommendations of the World Health Organisation (WHO) for trivalent vaccines in the Northern Hemisphere [19]. Although antigenically different, there has been shown some degree of cross-protection among both B lineages.

\section{Conclusion}

The GIHSN provides an opportunity to analyse influenza epidemiology and vaccine effectiveness worldwide.
In the $2016 / 17$ season, $\mathrm{A}(\mathrm{H} 3 \mathrm{~N} 2)$ was the predominant influenza strain this season (first wave), followed by B/ Victoria (second wave). Influenza $\mathrm{A}(\mathrm{H} 1 \mathrm{~N} 1) \mathrm{pdm} 09$ was mainly found in Mexico. A low vaccination coverage was seen in most of the GIHSN sites.

Differences in the distribution of influenza cases among the age groups were mainly due to the characteristics of the participating hospitals. Pregnant women had higher risk of testing positive for influenza, as occurred with diabetics, however this difference was not seen in COPD subjects.

Overall IVE was low to moderate 27.24 (95\% CI 15.62 to $37.27)$ in this season. A moderate to high effectiveness was seen for both influenza B lineages, and a non-significant low effectiveness for Influenza A(H1N1)pdm09.

\section{Additional file}

Additional file 1: Complementary Table S1. (DOCX $142 \mathrm{~kb}$ )
Abbreviations

AOR: Adjusted odds ratio; Cl: Confidence interval; GIHSN: Global Influenza Hospital Surveillance Network; IVE: Influenza vaccine effectiveness; OR: Odds ratio; RT-PCR: Reverse transcription-polymerase chain reaction 


\section{Acknowledgements}

The authors would like to acknowledge the Foundation for Influenza Epidemiology for the financial support and all members of the GIHSN, which are listed below (sites are firstly ordered by contribution to this manuscript and secondly by alphabetical order):

Valencia: B Escribano-López, S García Esteban, B Guglieri-López, M MartínNavarro, A Mira-Iglesias and M J Sánchez-Catalán from FISABIO-Salud Pública, Valencia, Spain, and X López-Labrador from FISABIO-Salud Pública, Valencia, Spain and the Consorcio de Investigación Biomédica de Epidemiología y Salud Pública, Spain, Instituto Carlos III, Madrid, Spain; E Adriana-Magos and M Carballido-Fernández from the Hospital General de Castellón, Castellón, Spain; J Mollar Maseres and M Roldán-Aguado from the Hospital Universitario y Politécnico La Fe, Valencia, Spain; J Fernández-Dopazo and M Tortajada-Girbés from the Hospital Doctor Peset, Valencia, Spain, and P Llorente-Nieto and G Schwarz-Chavarri from the Hospital General de Alicante, Alicante, Spain. Moscow: E Garina, L Kisteneva, L Kolobukhina, K Krasnoslobotsev, I Kruzhkova, L Merkulova and E Mukasheva from the D.I. Ivanovsky Institute of Virology FSBI "N.F. Gamaleya FRCEM" Ministry of Health, Moscow, Russian Federation. Canada: A Ambrose, M Andrew, M ElSherif, D MacKinnon-Cameron, M Nichols-Evans and P Ye from the Canadian Center for Vaccinology, IWK Health Centre and Nova Scotia Health Authority, Halifax, Canada. St. Petersburg: O Afanasieva, A Afanasieva, S Demina, E Dondurei, M Eropkin, A Fadeev, L Generalova, A Go, E Golovacheva, V Gonchar, A Komissarov, N Konovalova, S Kuvarzina, T Levanyuk, T Lobova, L Osidak, M Pisareva, E Rozhkova, K Sintsova, Z Sirotkina, E Smorodintseva, K Stolyarov, V Sukhovetskaya, M Tamila, L Voloshuk, M Yanina and P Zarishnyuk from the Research Institute of Influenza, St. Petersburg, Russian Federation. South Africa: S. A. Madhi from the Medical Research Council, Respiratory and Meningeal Pathogens Research Unit, University of the Witwatersrand, Johannesburg, South Africa.

Romania: V Aramă, D.Florea, M Luminos, D Otelea, O Sandulescu and O Vlaicu, from the National Institute of Infectious Diseases "Prof. Dr.MateiBals", Bucharest (INBIMB), Romania, and D Pitigoi from the National Institute of Infectious Diseases "Prof. Dr.MateiBals", Bucharest (INBIMB) and the University of Medicine and Pharmacy "Carol Davila" Bucharest, Romania. Turkey: K Aykac, T Bagcı Bosi, E Bilgin, M Durusu, A Kara, L Ozisik and S Tanir Basaranoglu from the Hacettepe University Faculty of Medicine, Ankara, Turkey; T Bedir Demirdag, O Guzel Tunccan, O Ozgen and H Tezer from the Gazi University Faculty of Medicine, Ankara, Turkey; B Gulhan and A OzkayaParlakay from the Ankara Hematology Oncology Children's Training and Research Hospital, Ankara, Turkey; M Ozsoy and N Tulek from the Ankara Research and Training Hospital, Ankara, Turkey, and M Akcay Ciblak, from Sanofi Pasteur, Turkey. Mexico: A Galindo Fraga, M L Guerrero Almeida and G M Ruiz-Palacios from the National Institute of Medical Sciences and Nutrition Salvador Zubirán (INCMNSZ), Mexico; A de Colsa Ranero and W Dolores Domínguez-Viveros from the Instituto Nacional de Pediatría, Mexico; I Jiménez-Escobar, J P Ramírez-Hinojosa and R P Vidal-Vázquez from the Hospital General Dr. Manuel Gea González, Mexico; D de la Rosa-Zamboni, A E Gamiño-Arroyo and S MorenoEspinosa from the Hospital Infantil de México, Mexico, and A Hernández from the Instituto Nacional de Enfermedades Infecciosas Ismael Cosio Villegas, Mexico. India: S Ali, M Khan, H Mir, Soumya and R Yusuf from the Sher-i-Kashmir Institute of Medical Sciences (SKIMS), India, and N Bali from the Department of Clinical Microbiology, Government Medical College, Srinagar, India. Czech Republic: M Havlickova, H Jirincova, R Kralova, Z Mandakova, J Prochazkova, H Sebestova from the National Institute of Public Health, Prague, Czech Republic, and D Dvorska, K Herrmanova, H Rohacova, T Rudova and I Standerova from the Hospital Na Bulovce, Prague, Czech Republic

Suzhou/Shanghai: K Chen, W Shan, F Zhang, G Zhao from the Fudan University, Shanghai, China; Y Yan from the Soochow University Affiliated Children Hospital, Suzhou, China; I Zheng from the Division of Infectious Disease, Key Laboratory of Surveillance and Early-warning on Infectious Disease, Chinese Center for Disease Control and Prevention, Beijing 102206, China, and J Pan from the State Key Laboratory of Respiratory Diseases, Guangzhou Institute of Respiratory Diseases, National Clinical Research Center for Respiratory Disease, The First Affiliated Hospital of Guangzhou Medical University, Guangzhou, Guangdong, China.

Kazakhstan: N Gaukhar from the Center for Sanitary-Epidemiological Expertise and Monitoring, Almaty, Kazakhstan.

Tunisia: S Amine from the Hôpital Charles-Nicolle, Tunis, Tunisia; J Ben Khelil from the Medical Intensive Care Unit, Abderrahmen Mami Hospital, Ariana,
Tunisia; M Ben Jeema and M Koubâa from the Hedi Chaker Hospital, Sfax, Tunisia; K Menif from the Children's Hospital of Tunis, Tunis, Tunisia; A Boukthir, S Chlif, M K Dellagi, A Gharbi, H Louzir, R Yazidi and W Zid from the Pasteur Institute of Tunis, Tunisia.

Peru: A Laguna, from the Instituto de Medicina Tropical Daniel Alcides Carrión, UNMSM, Lima, Peru; J Pérez-Bao, from the United States Naval Medical Research Center Detachment, lquitos and Lima, Peru, and N Reyes from the Universidad Nacional Mayor de San Marcos, Lima, Peru.

Ivory Coast: D Coulibaly, from the Pasteur Institute of Côte d'Ivoire, Abidjan, Côte d'Ivoire.

\section{Funding}

The study was funded by FISABIO-Public Health and the participating institutions of the manuscript (listed in the affiliations in the author list), and Sanofi Pasteur, who had no role in the analysis or discussion of the results. All participating institutions contributed to the data collection of the corresponding site, as well as the datasets transfer to FISABIO and the interpretation of GIHSN results. FISABIO-Public Health contributed to the design of the study, the recruitment and data collection of patients from Valencia Region and all participant sites, and the data analysis and interpretation of GIHSN results.

\section{Availability of data and materials}

Datasets were collected by each participating site and gathered on a pooled database by FISABIO. An authorisation is needed to any participating site in order to require sites databases. Data cannot be publicly shared due to confidentiality reasons, as some confidential patient data should not be shared, and in order to accomplish privacy laws from the participating sites. The corresponding author must be contacted with in order to ask for information about databases.

\section{Authors' contributions}

VBM wrote the manuscript and performed the statistical analysis. VBM, ST, SM, AS, MN, AD, SU, PK, JK, TZ, AK, ABS, EB, JDD, JPB (all authors) participated in the data collection, preparation and revision of the manuscript and approval of the final version and agreed with the common core protocol and the standard operating procedures of the GIHSN in order to keep the accuracy of the data.

\section{Ethics approval and consent to participate}

This study has been approved by the Ethics Committees of the participating sites, who have approved their participation in the GIHSN network. Each adult patient tested for influenza had signed an informed consent in order to be included in the study. In case the patient did not reach the legal age or is impaired, parents or legal guardians signed the informed consent. The Ethics Committees of the participating sites are listed below:

- St. Petersburg: Local Ethical Committee under the FGBU "Research Institute of Influenza" of the Ministry of Health of the Russian Federation

- Moscow: The local Ethic Committee of Hospital \#1 for Infectious Diseases of Moscow Health Department

- Kazakhstan: The study was carried in Almaty, Kazakhstan as part of the implementation of the national Severe Acute Respiratory Infections (SARI) surveillance program in Kazakhstan for purposes of communicable disease control. Ethical approval was not required but informed consent was obtained before inclusion. Informed consent provided in accordance with the Constitution of the Republic of Kazakhstan (section II article 29)

- Czech Republic: Ethics Committee of the Hospital Na Bulovce

- Canada: The Nova Scotia Health Authority Research Ethics Board and the IWK Research Ethics Board (IWK: Isaak Walton Killam)

- Romania: Bioethics Committee of the National Institute for Infectious Diseases "Prof. Dr. Matei Bals" Bucharest, Romania

- Turkey: Hacettepe University Non-interventional Clinical Research Ethics Board

- Valencia: Comité Ético de Investigación Clínica Dirección General de Salud Pública-Centro Superior de Investigación en Salud Pública (CEICDGSP-CSISP)

- Tunisia: The ethics committee of Abderrahmane Mami hospital, Ariana, Tunisia 
- Suzhou/Shanghai: Fudan University School of Public Health Institutional Review Board

- India: Institutional Ethics Committee of the Sher-i-Kashmir Institute of Medical Sciences, Srinagar

- Mexico: Research Ethics Committee of the National Institute of Medical Science and Nutrition Salvador Zubiran \& Research Committee of the National Institute of Medical Science and Nutrition Salvador Zubiran

- South Africa: The Human Research Ethics Committee of the University of the Witwatersrand

All of these Ethics Committees approved the participation of the site in the study and the data transfer to FISABIO, who led the implementation and data collection in the 2016-2017 season.

\section{Consent for publication}

Not applicable.

\section{Competing interests}

The authors declare that they have no competing interests.

\section{Publisher's Note}

Springer Nature remains neutral with regard to jurisdictional claims in published maps and institutional affiliations.

\section{Author details}

'Fundación para el Fomento de la Investigación Sanitaria y Biomédica de la Comunidad Valenciana (FISABIO), 21 Cataluña Av, 46020 Valencia, Spain. ${ }^{2}$ Ivanovsky Institute of Virology FSBI "N.F. Gamaleya FRCEM" Ministry of Health, Moscow, Russian Federation. ${ }^{3}$ Canadian Center for Vaccinology, IWK Health Centre and Nova Scotia Health Authority, Halifax, Canada. ${ }^{4}$ Research Institute of Influenza, WHO National Influenza Centre of Russia, St. Petersburg, Russian Federation. ${ }^{5}$ Medical Research Council, Respiratory and Meningeal Pathogens Research Unit, University of the Witwatersrand, Johannesburg, South Africa. ${ }^{6}$ Department of Science and Technology/ National Research Foundation, Vaccine Preventable Diseases, University of the Witwatersrand, Johannesburg, South Africa. ${ }^{7}$ National Institute of Infectious Diseases "Prof. Dr. Matei Bals", Bucharest (INBI "Prof. Dr. Matei Bals"), București, Romania. ${ }^{8}$ Turkish Society of Internal Medicine, Ankara, Turkey. ${ }^{9}$ Department of Internal and Pulmonary Medicine, Sher-i-Kashmir Institute of Medical Sciences (SKIMS), Soura, India. ${ }^{10}$ National Institute of Public Health, Prague, Czech Republic. ${ }^{11}$ Fudan University, Shanghai, China. ${ }^{12}$ Center for Sanitary-Epidemiological Expertise and Monitoring, Almaty, Kazakhstan.

${ }^{13}$ Pasteur Institute of Tunis, Tunis, Tunisia. ${ }^{14}$ College of Medicine and Medical Sciences, Manama, Bahrain.

Received: 7 September 2018 Accepted: 27 March 2019

Published online: 02 May 2019

\section{References}

1. Ortiz JR, Neuzil KM, Shay DK, Rue TC, Neradilek MB, Zhou H, et al. The burden of influenza-associated critical illness hospitalizations. Crit Care Med. 2014:42:2325-32

2. Ortiz JR, Neuzil KM, Rue TC, Zhou H, Shay DK, Cheng PY, et al. Population based incidence estimates of influenza-associated respiratory failure hospitalizations, 2003 to 2009. Am J Respir Crit Care Med. 2013;188:710-5.

3. Puig-Barbera J, Burtseva E, Yu H, Cowling BJ, Badur S, Kyncl J, et al. Influenza epidemiology and influenza vaccine effectiveness during the 2014-2015 season: annual report from the global influenza hospital surveillance network. BMC Public Health. 2016;16(Suppl 1):757. https://doi.org/10.1186/ s12889-016-3378-1.

4. Puig-Barberà J, Natividad-Sancho A, Trushakova S, Sominina A, Pisareva M, Ciblak MA, et al. Epidemiology of hospital admissions with influenza during the 2013/2014 northern hemisphere influenza season: results from the global influenza hospital surveillance network. PLoS One. 2016;11(5): e0154970. https://doi.org/10.1371/journal.pone.0154970.

5. Puig-Barberà J, Natividad-Sancho A, Launay O, Burtseva E, Ciblak MA, Tormos A, et al. 2012-2013 seasonal influenza vaccine effectiveness against influenza hospitalizations: results from the global influenza hospital surveillance network. PLoS One. 2014;9(6):e100497 https://doi.org/10.1371/ journal.pone.0100497.

6. Puig-Barberà, et al. First-year results of the global influenza hospital surveillance network: 2012 - 2013 northern hemisphere influenza season. BMC Public Health. 2014;14:564

7. Commission of the European Union. Official Journal of the European Union 27.9.2012. Influenza virus - Clinical criteria. L 262/16 (2012).

8. Sullivan SG, Tay EL, Kelly H. Variable definitions of the influenza season and their impact on vaccine effectiveness estimates. Vaccine. 2013;31:4280-3.

9. Puig-Barberà, et al. The global influenza hospital surveillance network (GIHSN): a new platform to describe the epidemiology of severe influenza. Influenza Other Respir Viruses. 2015;9(6):277-86.

10. Foppa IM, Haber M, Ferdinands JM, Shay DK. The case test-negative design for studies of the effectiveness of influenza vaccine. Vaccine. 2013:31:3104-9.

11. Kirkwood BR, Sterne JAC. Analysis of clustered data. In: Essential medical statistics. Malden: Blackwell Science; 2003. p. 355-70.

12. Higgins JP, Thompson SG. Quantifying heterogeneity in a meta-analysis. Stat Med. 2002;21:1539-58.

13. Higgins JP, Thompson SG, Deeks JJ, Altman DG. Measuring inconsistency in meta-analyses. BMJ. 2003;327:557-60

14. Caini Saverio, Alonso Wladimir J, Séblain Clotilde El-Guerche, Schellevis François, Paget John. The spatiotemporal characteristics of influenza a and B in the WHO European region: can one define influenza transmission zones in Europe?. Euro Surveill. 2017;22(35). https://doi.org/10.2807/1560-7917.ES. 2017.22.35.30606

15. Carrat F, Vergu E, Ferguson NM, Lemaitre M, Cauchemez S, Leach S, Valleron A-J. Time Lines of Infection and Disease in Human Influenza: A Review of Volunteer Challenge Studies. American journal of epidemiology. 2008; 167(Issue 7):775-85 https://doi.org/10.1093/aje/kwm375.

16. Dirección General de Salud Pública. Prevención y vigilancia de la gripe en la Comunitat Valenciana. Temporada 2016-2017. Available at: http:// publicaciones.san.gva.es/publicaciones/documentos/IS-150.pdf, last access 28 Nov 2018.

17. Esther K, Marc R, I-MOVE/I-MOVE+ study team. Early 2016/17 vaccine effectiveness estimates against influenza a(H3N2): I-MOVE multicentre case control studies at primary care and hospital levels in Europe. Euro Surveill. 2017;22(7) https://doi.org/10.2807/1560-7917.ES.2017.22.7.30464.

18. Centers for Disease Control and Prevention (CDC) (CDC). Estimated Influenza IIInesses, Medical visits, and Hospitalizations Averted by Vaccination in the United States. Available at: https://www.cdc.gov/flu/ about/disease/2016-17.htm, last access 6 Dec 2018.

19. World Health Organisation (WHO). Recommended composition of influenza virus vaccines for use in the 2016-2017 northern hemisphere influenza season. Available at: https://www.who.int/influenza/vaccines/virus/ recommendations/201602 recommendation.pdf?ua=1, last access 6 Dec 2018
Ready to submit your research? Choose BMC and benefit from:

- fast, convenient online submission

- thorough peer review by experienced researchers in your field

- rapid publication on acceptance

- support for research data, including large and complex data types

- gold Open Access which fosters wider collaboration and increased citations

- maximum visibility for your research: over $100 \mathrm{M}$ website views per year

At BMC, research is always in progress.

Learn more biomedcentral.com/submissions 